\title{
Some remarks on Toeplitz multipliers and Hankel matrices
}

\author{
by \\ Aleksander Pęczyński (Warszawa) and \\ Fyodor Sukochev (Bedford Park)
}

\begin{abstract}
Consider the set of all Toeplitz-Schur multipliers sending every upper triangular matrix from the trace class into a matrix with absolutely summable entries. We show that this set admits a description completely analogous to that of the set of all Fourier multipliers from $H_{1}$ into $\ell_{1}$. We characterize the set of all Schur multipliers sending matrices representing bounded operators on $\ell_{2}$ into matrices with absolutely summable entries. Next, we present a result (due to G. Pisier) that the upper triangular parts of such Schur multipliers are precisely the Schur multipliers sending upper triangular parts of matrices representing bounded linear operators on $\ell_{2}$ into matrices with absolutely summable entries. Finally, we complement solutions of Mazur's Problems 8 and 88 in the Scottish Book concerning Hankel matrices.
\end{abstract}

Introduction. In the present paper, we consider a few loosely connected questions concerning Toeplitz and Hankel matrices and a description of certain Schur multipliers acting on matrix representations of Schatten-von Neumann classes and their upper triangular subspaces. A Schur multiplier is a scalar matrix which acts by entrywise multiplication on some distinguished space of matrices. We are mainly interested in Schur multipliers with range consisting of matrices with absolutely summable entries. We show that (cf. Theorem 3.4) descriptions of certain classes of Schur multipliers, in particular, those induced by Toeplitz matrices are similar to the corresponding descriptions of Fourier multipliers. In Section 3, we show that Schur multipliers induced by Toeplitz matrices which map the upper triangular trace class $\mathcal{S}_{1}$ into absolutely summable matrices are determined by the same class of scalar sequences as the Fourier multipliers from the Hardy space $H_{1}$ into $\ell_{1}$ described by Ch. Fefferman ([SlSt], [SzW]). Fefferman's description plays an important role in our argument. The paradigm for Theorem 3.4 is the result of A. Shields [Sh] who proved the result in the case of the upper triangular part of the Hilbert matrix.

2000 Mathematics Subject Classification: 47B35, 47B49, 15A60, 47B10.

Key words and phrases: upper triangular matrices, Toeplitz matrices, Hankel matrices, Schur and Toeplitz-Schur multipliers, trace classes.

Research is partially supported by the ARC. 
Sections 4 and 5 deal with Schur multipliers from $B\left(\ell_{2}\right)$ and from the upper triangular part of $B\left(\ell_{2}\right)$ respectively. Theorem 4.1 says that the space of Schur multipliers which map $B\left(\ell_{2}\right)$ into absolutely summable matrices is dual to the space of matrices which, when multiplied by a suitable matrix of signs, represent a bounded linear operator on $\ell_{2}$. Our result is a relatively easy consequence of a deep result of Lust-Piquard [Lu] which is a non-commutative analogue of the result of [KKL] on Fourier coefficients of continuous functions. Theorem 5.1 (due to Pisier) says that the upper triangular subspace of the space of multipliers described in Theorem 4.1 consists of all Schur multipliers from the upper triangular subspace of $B\left(\ell_{2}\right)$ into the space of absolutely summable matrices. The proof is based on Pisier's theory of $C^{*}$-summing operators and on one of his interpolation theorems. Theorems 4.2 and 5.1 are in full analogy with the classical descriptions of Fourier multipliers from the space of continuous functions and from the disc algebra to $\ell_{1}$.

Section 6 deals with Hankel matrices. We present sharp quantitative answers to Mazur's Problems 8 and 88 from the Scottish Book. The problems have been solved independently by Eggermont and Leung, and by Kwapien and the first named author [EL], [KwP].

Acknowledgements. The authors are grateful to Professor Gilles Pisier for his permission to publish Theorem 5.1 in this paper. We are indebted to Professor Françoise Lust-Piquard who read earlier versions of the paper and suggested many improvements. Also we would like to thank Professor Stanisław Kwapień who allowed us to use an idea from the unpublished lecture $[\mathrm{KwP}]$.

1. Preliminaries and notation. $\mathcal{M}$ stands for the space of all com-

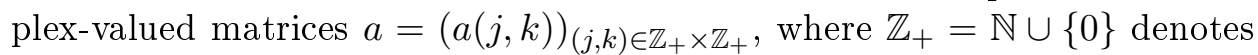
the non-negative integers. $\mathcal{M}_{0}$ stands for the space of all matrices $a \in \mathcal{M}$ with finite support, where $\operatorname{supp} a=\{(j, k): a(j, k) \neq 0\}$. We consider the (quasi) Banach spaces of matrices

where

$$
\mathcal{M}_{p}=\left\{a \in \mathcal{M}:\|a\|_{\mathcal{M}_{p}}<\infty\right\}
$$

$$
\|a\|_{\mathcal{M}_{p}}=\|a\|_{\ell_{p}\left(\mathbb{Z}_{+} \times \mathbb{Z}_{+}\right)}= \begin{cases}\left(\sum_{(j, k) \in \mathbb{Z}_{+} \times \mathbb{Z}_{+}}|a(j, k)|^{p}\right)^{1 / p} & \text { for } 0<p<\infty, \\ \sup _{(j, k) \in \mathbb{Z}_{+} \times \mathbb{Z}_{+}}|a(j, k)| & \text { for } p=\infty .\end{cases}
$$

$B\left(\ell_{2}\right)$ denotes the algebra of all bounded operators on $\ell_{2}=\ell_{2}\left(\mathbb{Z}_{+}\right)$, with the usual multiplication of matrices $c=a b$, where

Hence

$$
c(j, k)=\sum_{s \in \mathbb{Z}_{+}} a(j, s) b(s, k) \quad \text { for } j, k \in \mathbb{Z}_{+} .
$$

$$
B\left(\ell_{2}\right)=\left\{a \in \mathcal{M}:\|a\|_{B\left(\ell_{2}\right)}<\infty\right\},
$$


where

$$
\|a\|_{B\left(\ell_{2}\right)}=\left\|a: \ell_{2} \rightarrow \ell_{2}\right\|=\sup _{\sum_{k}\left|\xi_{k}\right|^{2}=1}\left(\sum_{j \in \mathbb{Z}_{+}}\left|\sum_{k \in \mathbb{Z}_{+}} a(j, k) \xi_{k}\right|^{2}\right)^{1 / 2} ;
$$

$K\left(\ell_{2}\right)$ denotes the subspace of $B\left(\ell_{2}\right)$ of all compact operators, hence

$$
K\left(\ell_{2}\right)=\left\{a \in B\left(\ell_{2}\right): \lim _{j, k \rightarrow \infty} a(j, k)=0\right\} .
$$

We set

$$
\mathcal{S}_{p}=\left\{a \in \mathcal{M}:\|a\|_{p}<\infty\right\} \quad \text { for } 0<p<\infty,
$$

where $\|a\|_{p}=\left(\operatorname{Tr}\left(\left|a^{*} a\right|^{p / 2}\right)\right)^{1 / p}, a^{*}$ is the adjoint of $a$, i.e. $a^{*}(j, k)=\overline{a(k, j)}$ for $j, k \in \mathbb{Z}_{+}$. By $|a|=\left(a^{*} a\right)^{1 / 2}$ we denote the modulus of $a$, and Tr stands for the usual trace, i.e. $\operatorname{Tr}(a)=\sum_{j \in \mathbb{Z}_{+}} a(j, j)$.

The spaces $\mathcal{S}_{p}$ are called the Schatten classes; $\mathcal{S}_{1}$ can be identified with the nuclear operators on $\ell_{2}$, and $\mathcal{S}_{2}$ with the Hilbert-Schmidt operators.

Besides $|a|$ and $a^{*}$, we also consider the matrix of absolute values $a^{\natural}$ and the transposed matrix ${ }^{t} a$ defined by

$$
a^{\natural}(j, k)=|a(j, k)|, \quad{ }^{t} a(j, k)=a(k, j) \quad\left((j, k) \in \mathbb{Z}_{+} \times \mathbb{Z}_{+}\right) .
$$

We write $\varepsilon^{\natural} \equiv 1$ whenever $\varepsilon \in \mathcal{M}$ satisfies $|\varepsilon(j, k)|=1$ for all $(j, k) \in \mathbb{Z}_{+} \times \mathbb{Z}_{+}$.

An element $a \in \mathcal{M}$ is upper triangular (resp. lower triangular), in symbols $a \in \mathcal{U T}$ (resp. $a \in \mathcal{L} \mathcal{T}$ ), provided that $a(j, k)=0$ for $j>k$ (resp. $a(j, k)=0$ for $j<k$ ). If $X$ is a space of matrices then we put

$$
\mathcal{U} \mathcal{T} X:=\{a \in X: a \in \mathcal{U} \mathcal{T}\}, \quad \mathcal{L} \mathcal{T} X:=\{a \in X: a \in \mathcal{L} \mathcal{T}\}
$$

A matrix $b \in \mathcal{M}$ is an extension of an $a \in \mathcal{U T}$ (resp. $a \in \mathcal{L} \mathcal{T}$ ) provided that $a(j, k)=b(j, k)$ for $j \leq k$ (resp. $a(j, k)=b(j, k)$ for $j \geq k)$.

A matrix which is constant on all the diagonals parallel to the main diagonal is called a Toeplitz matrix, i.e. $a \in \mathcal{M}$ is Toeplitz if and only if there exists a sequence $\left(m_{s}\right)_{s \in \mathbb{Z}}$ such that $a(j, k)=m_{k-j}$ for $j, k \in \mathbb{Z}_{+}$. If $a \in \mathcal{U T}$ is Toeplitz then it is determined by the sequence $\left(m_{s}\right)_{s \in \mathbb{Z}_{+}}$.

The Schur product of $a, b \in \mathcal{M}$ is the matrix $a \diamond b$ defined by

$$
a \diamond b(j, k)=a(j, k) b(j, k) \quad \text { for } j, k \in \mathbb{Z}_{+} .
$$

The Schur multiplier induced by an $a \in \mathcal{M}$ is the operator $S_{a}: \mathcal{M} \rightarrow \mathcal{M}$ defined by $S_{a}(b)=a \diamond b$. A Toeplitz multiplier is a Schur multiplier induced by a Toeplitz matrix. If $a$ is a Toeplitz matrix determined by a sequence $m$ then the induced Toeplitz multiplier is usually denoted by $T_{m}$. Clearly Schur multipliers preserve the class of upper triangular (resp. lower triangular) matrices. If $X$ and $Y$ are spaces of matrices then the space of all Schur multipliers $X$ into $Y$ is denoted by $\mathbb{S}(X, Y)$. 
The trace duality is determined by the bilinear form

$$
(a, b) \mapsto \operatorname{Tr}(b a)=\sum_{j, k \in \mathbb{Z}_{+}} a(j, k) b(k, j)
$$

defined whenever the right hand side makes sense.

2. Schur multipliers from $\mathcal{U} \mathcal{T} \mathcal{S}_{1}$ into $\mathcal{M}_{1}$. In Proposition 2.1 below, we describe the Schur multipliers from $\mathcal{S}_{1}$ into $\mathcal{M}_{1}$. This is a folklore result, e.g. it is implicitly stated without proof in [DLuQ].

For a scalar sequence $x=\left(\xi_{j}\right)_{j \in \mathbb{Z}_{+}}$we put $x^{\natural}=\left(\left|\xi_{j}\right|\right)_{j \in \mathbb{Z}_{+}}$. For $x=$ $\left(\xi_{j}\right)_{j \in \mathbb{Z}_{+}}$and $y=\left(\eta_{k}\right)_{k \in \mathbb{Z}_{+}}$define the simple tensor $x \otimes y \in \mathcal{M}$ by $x \otimes y(j, k)=$ $\xi_{j} \eta_{k}$ for $j, k \in \mathbb{Z}_{+}$. We denote by $\langle\cdot, \cdot\rangle$ the usual scalar product in $\ell_{2}$.

Proposition 2.1. The Schur multiplier $S_{b}$ is a bounded linear operator from $\mathcal{S}_{1}$ into $\mathcal{M}_{1}$ if and only if $b^{\natural} \in B\left(\ell_{2}\right)$. Moreover $\left\|S_{b}: \mathcal{S}_{1} \rightarrow \mathcal{M}_{1}\right\|$ $=\left\|b^{\natural}\right\|_{B\left(\ell_{2}\right)}$.

Proof. Recall that the extreme points of the unit ball of $\mathcal{S}_{1}$ are all the simple tensors $x \otimes y$ with $\|x\|_{\ell_{2}}\|y\|_{\ell_{2}}=1$; moreover, convex combinations of the extreme points are norm dense in the unit ball of $\mathcal{S}_{1}$. Thus, we have

$$
\begin{aligned}
\left\|S_{b}: \mathcal{S}_{1} \rightarrow \mathcal{M}_{1}\right\| & =\sup _{\|x\|_{\ell_{2}}=\|y\|_{\ell_{2}}=1}\left\|S_{b}(x \otimes y)\right\|_{\mathcal{M}_{1}} \\
& =\sup _{\|x\|_{\ell_{2}}=\|y\|_{\ell_{2}}=1}\|b \diamond x \otimes y\|_{\mathcal{M}_{1}} \\
& =\sup _{\|x\|_{\ell_{2}}=\|y\|_{\ell_{2}=1}}\left\|b^{\natural} \diamond x^{\natural} \otimes y^{\natural}\right\|_{\mathcal{M}_{1}} \\
& =\sup _{\|x\|_{\ell_{2}}=\|y\|_{\ell_{2}}=1}\left\langle b^{\natural}\left(x^{\natural}\right), y^{\natural}\right\rangle=\left\|b^{\natural}\right\|_{B\left(\ell_{2}\right)} .
\end{aligned}
$$

The main result of this section (Proposition 2.2), which describes the Schur multipliers from $\mathcal{U} \mathcal{T} \mathcal{S}_{1}$ into $\mathcal{M}_{1}$, is an easy consequence of the following "Arveson Criterion" (cf. [A], [Ov]). For $a \in \mathcal{L} \mathcal{T}$ and $s \in \mathbb{Z}_{+}$, define $Q_{s}(a) \in \mathcal{L} \mathcal{T}$ by

$$
Q_{s}(a)(j, k)= \begin{cases}a(j, k) & \text { for } 0 \leq k \leq s, s \leq j<\infty \\ 0 & \text { otherwise }\end{cases}
$$

Arveson Criterion. An element $a \in \mathcal{L} \mathcal{T}$ admits an extension to an element $c \in B\left(\ell_{2}\right)$ if and only if

$$
\sup _{s \in \mathbb{Z}_{+}}\left\|Q_{s}(a)\right\|_{B\left(\ell_{2}\right)}<\infty
$$

Moreover, if (2.1) holds, then there exists an extension $c \in B\left(\ell_{2}\right)$ of a such that

$$
\|c\|_{B\left(\ell_{2}\right)}=\sup _{s \in \mathbb{Z}_{+}}\left\|Q_{s}(a)\right\|_{B\left(\ell_{2}\right)} .
$$

We are ready to state 
Proposition 2.2. Let $b \in \mathcal{U} \mathcal{T}$. The Schur multiplier $S_{b}$ takes $\mathcal{U} \mathcal{T} \mathcal{S}_{1}$ into $\mathcal{M}_{1}$ if and only if

$$
\sup _{s \in \mathbb{Z}_{+}}\left\|Q_{s}\left({ }^{t} b^{\natural}\right)\right\|_{B\left(\ell_{2}\right)}<\infty .
$$

Moreover if (2.2) holds then $S_{b}$ is a bounded linear operator from $\mathcal{U} \mathcal{T} \mathcal{S}_{1}$ into $\mathcal{M}_{1}$ and

$$
\left\|S_{b}: \mathcal{U} \mathcal{T} \mathcal{S}_{1} \rightarrow \mathcal{M}_{1}\right\|=\sup _{s \in \mathbb{Z}_{+}}\left\|Q_{s}\left({ }^{t} b^{\natural}\right)\right\|_{B\left(\ell_{2}\right)} .
$$

Proof. Assume that $S_{b}\left(\mathcal{U} \mathcal{T} \mathcal{S}_{1}\right) \subseteq \mathcal{M}_{1}$. Since the matrices in $\mathcal{U} \mathcal{T} \mathcal{S}_{1}$ with finitely many non-zero entries are dense in $\mathcal{U} \mathcal{T} \mathcal{S}_{1}$, a routine application of the Banach-Steinhaus principle implies that $S_{b}$ is a bounded linear operator from $\mathcal{U} \mathcal{T} \mathcal{S}_{1}$ into $\mathcal{M}_{1}$. Let us put

$$
a^{*}(x)=\sum_{k \in \mathbb{Z}_{+}} \sum_{j=0}^{k} b^{\natural}(j, k) x(j, k) \quad \text { for } x=(x(j, k)) \in \mathcal{U} \mathcal{T} \mathcal{S}_{1} .
$$

Clearly

$$
\left|a^{*}(x)\right| \leq \sum_{k \in \mathbb{Z}_{+}} \sum_{j=0}^{k}|b(j, k) x(j, k)|=\left\|S_{b}(x)\right\|_{\mathcal{M}_{1}} \leq\left\|S_{b}: \mathcal{U} \mathcal{T} \mathcal{S}_{1} \rightarrow \mathcal{M}_{1}\right\|\|x\|_{1} .
$$

Thus $a^{*}$ is a bounded linear functional on $\mathcal{U} \mathcal{T} \mathcal{S}_{1}$ with $\left\|a^{*}\right\| \leq \| S_{b}$ : $\mathcal{U} \mathcal{T}_{1} \rightarrow \mathcal{M}_{1} \|$. Let $\widetilde{a}^{*}$ be the norm preserving extension of $a^{*}$ to a linear functional on $\mathcal{S}_{1}$. Then in the trace duality $\widetilde{a}^{*}$ is represented by a matrix $c \in B\left(\ell_{2}\right)$ such that $\|c\|_{B\left(\ell_{2}\right)} \leq\left\|S_{b}: \mathcal{U} \mathcal{T} \mathcal{S}_{1} \rightarrow \mathcal{M}_{1}\right\|$ and $c(k, j)=b^{\natural}(j, k)$ for $0 \leq j \leq k, k \in \mathbb{Z}_{+}$. Hence $c$ is an extension of ${ }^{t} b^{\natural}$. By the Arveson Criterion, $\sup _{s \in \mathbb{Z}_{+}}\left\|Q_{s}\left({ }^{t} b^{\natural}\right)\right\|_{B\left(\ell_{2}\right)} \leq\left\|S_{b}: \mathcal{U} \mathcal{T} \mathcal{S}_{1} \rightarrow \mathcal{M}_{1}\right\|$.

Conversely, assume (2.2). Fix $\varepsilon \in \mathcal{M}$ with $\varepsilon^{\natural} \equiv 1$. Note that

$$
\sup _{s \in \mathbb{Z}_{+}}\left\|Q_{s}\left({ }^{t}(\varepsilon \diamond b)\right)\right\|_{B\left(\ell_{2}\right)} \leq \sup _{s \in \mathbb{Z}_{+}}\left\|Q\left({ }^{t} b^{\natural}\right)\right\|_{B\left(\ell_{2}\right)}
$$

(because for every $a \in B\left(\ell_{2}\right)$ one has $\|\varepsilon \diamond a\|_{B\left(\ell_{2}\right)} \leq\left\|a^{\natural}\right\|_{B\left(\ell_{2}\right)}$ ). It follows from (2.2) and (2.3) combined with the Arveson Criterion that there exists $c_{\varepsilon}$ which extends ${ }^{t}(\varepsilon \diamond b)$ and satisfies $\left\|c_{\varepsilon}\right\|_{B\left(\ell_{2}\right)} \leq \sup _{s \in \mathbb{Z}_{+}}\left\|Q_{s}\left({ }^{t} b^{\natural}\right)\right\|_{B\left(\ell_{2}\right)}$. Thus

$$
\operatorname{Tr}\left({ }^{t} c_{\varepsilon} x\right)=\sum_{k \in \mathbb{Z}_{+}} \sum_{j=0}^{k} \varepsilon(j, k) b(j, k) x(j, k) \quad\left(x=(x(j, k)) \in \mathcal{U} \mathcal{T} \mathcal{S}_{1}\right) .
$$

Therefore, in view of the trace duality,

$$
\left\|S_{b}(x)\right\|_{\mathcal{M}_{1}}=\sup _{\varepsilon^{\natural} \equiv 1}\left|\sum_{k \in \mathbb{Z}_{+}} \sum_{j=0}^{k} \varepsilon(j, k) b(j, k) x(j, k)\right| \leq \sup _{\varepsilon^{\natural} \equiv 1}\left\|c_{\varepsilon}\right\|_{B\left(\ell_{2}\right)}\|x\|_{1} .
$$


Hence

$$
\left\|S_{b}: \mathcal{U T} \mathcal{S}_{1} \rightarrow \mathcal{M}_{1}\right\| \leq \sup _{\varepsilon^{\natural} \equiv 1}\left\|c_{\varepsilon}\right\|_{B\left(\ell_{2}\right)} \leq \sup _{s \in \mathbb{Z}_{+}}\left\|Q_{s}\left({ }^{t} b^{\natural}\right)\right\|_{B\left(\ell_{2}\right)} .
$$

REMARK 2.3. A particular case of Proposition 2.2 is [Sh, Theorem 2b] on matrices with positive entries.

3. Toeplitz multipliers from $\mathcal{U} \mathcal{T} \mathcal{S}_{1}$ into $\mathcal{M}_{1}$. Our first result is well known: it gives a satisfactory description of Toeplitz multipliers from $\mathcal{S}_{1}$ into $\mathcal{M}_{1}$. As we learned from Prof. F. Lust-Piquard, it goes back to [Dg, Bn] (after reformulating it in the languague of the Fourier transform).

Proposition 3.1. A Toeplitz multiplier $T_{m}$ is a bounded linear operator from $\mathcal{S}_{1}$ into $\mathcal{M}_{1}$ if and only if $m=\left(m_{s}\right)_{s \in \mathbb{Z}} \in \ell_{1}(\mathbb{Z})$. Moreover

$$
\left\|T_{m}: \mathcal{S}_{1} \rightarrow \mathcal{M}_{1}\right\|=\|m\|_{\ell_{1}(\mathbb{Z})} \text {. }
$$

Proof. To prove that $T_{m}$ with $m \in \ell_{1}(\mathbb{Z})$ is bounded it is enough to verify its action on the extreme points of the unit ball $B_{\mathcal{S}_{1}}$ of $\mathcal{S}_{1}$. Let $a=x \otimes y$ with $x=\left(\xi_{j}\right)_{j \in \mathbb{Z}_{+}}, y=\left(\eta_{k}\right)_{k \in \mathbb{Z}_{+}}$and $\|x\|_{\ell_{2}}\|y\|_{\ell_{2}}=1$ be an extreme point of $B_{\mathcal{S}_{1}}$. We have

$$
\begin{aligned}
\left\|T_{m}(x \otimes y)\right\|_{\mathcal{M}_{1}}= & \sum_{j, k \in \mathbb{Z}_{+}}\left|m_{j-k}\right|\left|\xi_{j}\right|\left|\eta_{k}\right| \\
= & \sum_{s \geq 0} \sum_{j=0}^{\infty}\left|m_{s}\right|\left|\xi_{j}\right|\left|\eta_{j+s}\right|+\sum_{s<0}\left|m_{s}\right| \sum_{k=0}^{\infty}\left|\xi_{k-s}\right|\left|\eta_{k}\right| \\
\leq & \sum_{s \geq 0}\left|m_{s}\right|\left(\sum_{j=0}^{\infty}\left|\xi_{j}\right|^{2}\right)^{1 / 2}\left(\sum_{k=s}^{\infty}\left|\eta_{k}\right|^{2}\right)^{1 / 2} \\
& +\sum_{s<0}\left|m_{s}\right|\left(\sum_{j=-s}^{\infty}\left|\xi_{j}\right|^{2}\right)^{1 / 2}\left(\sum_{k=0}^{\infty}\left|\eta_{k}\right|^{2}\right)^{1 / 2} \\
\leq & \sum_{s \in \mathbb{Z}}\left|m_{s}\right|\|x\|_{\ell_{2}}\|y\|_{\ell_{2}}=\sum_{s \in \mathbb{Z}}\left|m_{s}\right|=\|m\|_{\ell_{1}(\mathbb{Z})}
\end{aligned}
$$

Thus $\left\|T_{m}: \mathcal{S}_{1} \rightarrow \mathcal{M}_{1}\right\| \leq\|m\|_{\ell_{1}(\mathbb{Z})}$. Conversely, let $a^{(n)}=x^{(n)} \otimes x^{(n)}$, where

$$
x^{(n)}=(\underbrace{(n+1)^{-1 / 2}, \ldots,(n+1)^{-1 / 2}}_{n+1}, 0,0, \ldots) \text {. }
$$

Then $\left\|a^{(n)}\right\|_{1}=1$ while

$$
\left\|T_{m}\left(a^{(n)}\right)\right\|_{\mathcal{M}_{1}}=\sum_{s=-n}^{n}\left|m_{s}\right| \frac{n+1-|s|}{n+1} .
$$

Thus

$$
\left\|T_{m}: \mathcal{S}_{1} \rightarrow \mathcal{M}_{1}\right\| \geq \lim _{n}\left\|T_{m}\left(a^{(n)}\right)\right\|_{\mathcal{M}_{1}}=\|m\|_{\ell_{1}(\mathbb{Z})}
$$


Next, we discuss Toeplitz multipliers from $\mathcal{U} \mathcal{T} \mathcal{S}_{1}$ into $\mathcal{M}_{1}$.

For a complex sequence $m=\left(m_{s}\right)_{s \in \mathbb{Z}_{+}}$we put

$$
\varrho(m)=\left(\left|m_{0}\right|^{2}+\left|m_{1}\right|^{2}+\sup _{r \geq 1} \sum_{q=1}^{\infty}\left(\sum_{s=r q+1}^{r(q+1)}\left|m_{s}\right|\right)^{2}\right)^{1 / 2} .
$$

We write $m \in$ FM if and only if $\varrho(m)<\infty$. Here FM stands for "Fefferman multipliers"; in [BP] they are called $H_{1}-\ell_{1}$ multipliers. Recall that about 1975 Charles Fefferman discovered that the condition $m \in \mathrm{FM}$ characterizes the Fourier multipliers from the Hardy space $H_{1}$ on the unit disc into $\ell_{1}$. For the proof see [SzW, SlSt]. It was observed in [BP] that Fefferman's result generalizes to $\mathcal{S}_{1}$-valued $H_{1}$ as follows:

THEOREM 3.2. For a complex sequence $m=\left(m_{s}\right)_{s \in \mathbb{Z}_{+}}$the following are equivalent:

(i) $m \in \mathrm{FM}$,

(ii) there is a constant $c>0$ independent of $m$ such that

$$
\sum_{s \in \mathbb{Z}_{+}}\left|m_{s}\right|\|\widehat{f}(s)\|_{1} \leq c \varrho(m)(2 \pi)^{-1} \int_{-\pi}^{\pi}\left\|f\left(e^{i t}\right)\right\|_{1} d t \quad \text { for } f \in H_{1}\left(\mathbb{T} ; \mathcal{S}_{1}\right) .
$$

Here, $H_{1}\left(\mathbb{T} ; \mathcal{S}_{1}\right)$ denotes the closure in the norm $(2 \pi)^{-1} \int_{-\pi}^{\pi}\left\|f\left(e^{i t}\right)\right\|_{\mathcal{S}_{1}} d t$ of the linear span of "monomials" $\left\{t \mapsto e^{i s t} a: s \in \mathbb{Z}_{+}, a \in \mathcal{S}_{1}\right\}$, and the Fourier coefficients are defined by $\widehat{f}(s)=(2 \pi)^{-1} \int_{-\pi}^{\pi} f\left(e^{i t}\right) e^{-i s t} d t$ for $f \in$ $H_{1}\left(\mathbb{T} ; \mathcal{S}_{1}\right), s \in \mathbb{Z}_{+}$.

Our next result can be regarded as the non-commutative analogue of the original Fefferman characterization of the Fourier multipliers from $H_{1}$ into $\ell_{1}$.

TheOREM 3.3. A complex sequence $m=\left(m_{s}\right)_{s \in \mathbb{Z}_{+}}$is in FM if and only if the Toeplitz multiplier $T_{m}$ maps $\mathcal{U} \mathcal{T} \mathcal{S}_{1}$ into $\mathcal{M}_{1}$. Precisely, the following inequality holds:

$$
(\sqrt{3})^{-1} \varrho(m) \leq\left\|T_{m}: \mathcal{U} \mathcal{T} \mathcal{S}_{1} \rightarrow \mathcal{M}_{1}\right\| \leq c \varrho(m) .
$$

The constant $c$ in (3.1) is the same as in Theorem 3.2 as well as in the original Fefferman inequality for scalar-valued functions.

Proof. Since the eventually zero matrices are dense in $\mathcal{U} \mathcal{T} \mathcal{S}_{1}$, the Banach-Steinhaus theorem implies that if $T_{m}\left(\mathcal{U} \mathcal{T} \mathcal{S}_{1}\right) \subset \mathcal{M}_{1}$ then $T_{m}$ is a bounded operator. Thus, it is enough to establish (3.1) for eventually zero matrices. 
The right hand inequality of (3.1). For $a \in \mathcal{U} \mathcal{T} \mathcal{S}_{1}$ and $s \in \mathbb{Z}_{+}$, define the " $s$-diagonal" matrix $d_{s}(a)$ by

$$
d_{s}(a)(j, k)= \begin{cases}a(j, k) & \text { for } k-j=s \\ 0 & \text { otherwise. }\end{cases}
$$

Clearly $d_{s}(a) \in \mathcal{S}_{1}$ and $\left\|d_{s}(a)\right\|_{1}=\sum_{k=s}^{\infty}|a(k-s, k)|$. Consider the $\mathcal{S}_{1}$-valued function

$$
f\left(e^{i t}\right)=\sum_{s=0}^{\infty} d_{s}(a) \exp (i s t) \quad(-\pi \leq t \leq \pi) .
$$

We have the identity (cf. [DoS])

$$
f\left(e^{i t}\right)=u_{-t} a u_{t} \quad(-\pi \leq t \leq \pi)
$$

where $u_{t}$ is the unitary diagonal matrix defined by

$$
u_{t}(j, k)= \begin{cases}\exp (i j t) & \text { for } j=k \\ 0 & \text { for } j \neq k\end{cases}
$$

Remembering that $u_{t}$ is unitary, it follows from (3.3) that

$$
\left\|f\left(e^{i t}\right)\right\|_{1}=\left\|u_{t}\right\|_{B\left(\ell_{2}\right)}\|a\|_{1}\left\|u_{-t}\right\|_{B\left(\ell_{2}\right)}=\|a\|_{1} .
$$

Thus,

$$
(2 \pi)^{-1} \int_{-\pi}^{\pi}\left\|f\left(e^{i t}\right)\right\|_{1} d t=\|a\|_{1} .
$$

Hence, $f \in H_{1}\left(\mathbb{T} ; \mathcal{S}_{1}\right)$; obviously $\widehat{f}(s)=d_{s}(a)$ for $s \in \mathbb{Z}_{+}$. Thus, by Theorem 3.2 ,

$$
\sum_{s=0}^{\infty}\|\widehat{f}(s)\|_{1}\left|m_{s}\right| \leq c \varrho(m)(2 \pi)^{-1} \int_{-\pi}^{\pi}\left\|f\left(e^{i t}\right)\right\|_{1} d t .
$$

Hence, invoking (3.4), we obtain

$$
\begin{aligned}
\left\|T_{m}(a)\right\|_{\mathcal{M}_{1}} & =\sum_{k=0}^{\infty} \sum_{j=0}^{k}\left|a(j, k) m_{k-j}\right|=\sum_{s=0}^{\infty}\left|m_{s}\right| \sum_{k=s}^{\infty}|a(k-s, k)| \\
& =\sum_{s=0}^{\infty}\left|m_{s}\right|\left\|d_{s}(a)\right\|_{1} \leq c \varrho(m)\|a\|_{1} .
\end{aligned}
$$

Therefore, $\left\|T_{m}: \mathcal{U} \mathcal{T} \mathcal{S}_{1} \rightarrow \mathcal{M}_{1}\right\| \leq c \varrho(m)$, which completes the proof of the right hand inequality of (3.1) .

The left hand inequality of (3.1). Assume that $\left\|T_{m}: \mathcal{U} \mathcal{T} \mathcal{S}_{1} \rightarrow \mathcal{M}_{1}\right\|<\infty$. Without loss of generality we may assume that $m_{s} \geq 0$ for $s \in \mathbb{Z}_{+}$. Fix a positive integer $r$ and an eventually zero non-negative sequence $\left(\alpha_{q}\right)_{q \in \mathbb{Z}_{+}}$ 
with $\sum_{q=0}^{\infty} \alpha_{q}^{2}=1$. Define upper triangular matrices $a, a_{1}, a_{2}$ by

$$
\begin{aligned}
a & =a_{1}+a_{2}, \\
a_{1} & =x \otimes y, \quad x=(1,0,0, \ldots), y=\left(\alpha_{0}, \alpha_{1}, 0,0, \ldots\right), \\
a_{2} & =u \otimes v, \quad u=(\underbrace{r^{-1 / 2}, \ldots, r^{-1 / 2}}_{r}, 0,0, \ldots), v=\left(v_{k}\right)_{k \in \mathbb{Z}_{+}},
\end{aligned}
$$

where

$$
v_{k}= \begin{cases}0 & \text { for } 0 \leq k \leq r \\ r^{-1 / 2} \alpha_{2} & \text { for } r+1 \leq k \leq 2 r \\ r^{-1 / 2} \max \left(\alpha_{q}, \alpha_{q+1}\right) & \text { for } r q+1 \leq k \leq r(q+1)(q=2,3, \ldots) .\end{cases}
$$

Put $t=\alpha_{0}^{2}+\alpha_{1}^{2}$. Clearly $\|x\|_{\ell_{2}}=1$ and $\|y\|_{\ell_{2}}=\sqrt{t}$. Thus $\left\|a_{1}\right\|_{1}=\sqrt{t}$. Similarly $\|u\|_{\ell_{2}}=1$ and

$$
\|v\|_{\ell_{2}}=\left(\alpha_{2}^{2}+\sum_{q=1}^{\infty} \max \left(\alpha_{q+1}, \alpha_{q+2}\right)^{2}\right)^{1 / 2} \leq \sqrt{2}\left(\sum_{q=2}^{\infty} \alpha_{q}^{2}\right)^{1 / 2}=\sqrt{2(1-t)} .
$$

Thus, $\left\|a_{2}\right\|_{1} \leq \sqrt{2(1-t)}$. Hence,

$$
\|a\|_{1} \leq\left\|a_{1}\right\|_{1}+\left\|a_{2}\right\|_{1} \leq \sqrt{t}+\sqrt{2(1-t)} \leq \sqrt{3},
$$

because the function $t \mapsto \sqrt{t}+\sqrt{2(1-t)}$ for $0 \leq t \leq 1$ attains its maximum at $t=1 / 3$. Therefore

$$
\sqrt{3}\left\|T_{m}: \mathcal{U} \mathcal{T} \mathcal{S}_{1} \rightarrow \mathcal{M}_{1}\right\| \geq\left\|T_{m}(a)\right\|_{\mathcal{M}_{1}} .
$$

Clearly, the entries of $a$ satisfy

$$
a(j, k)= \begin{cases}\alpha_{0} & \text { for } j=0, k=0 \\ \alpha_{1} & \text { for } j=0, k=1 \\ \alpha_{2} / r & \text { for } 0 \leq j<r, r+1 \leq k \leq 2 r \\ \max \left(\alpha_{q}, \alpha_{q+1}\right) / r & \text { for } 0 \leq j<r, r q+1 \leq k \leq r(q+1)(q \geq 2), \\ 0 & \text { otherwise. }\end{cases}
$$

Obviously, $T_{m}(a)$ satisfies

$$
T_{m}(a)(j, k)=a(j, k) m_{k-j} \quad \text { for } 0 \leq j \leq k \text { and } k \in \mathbb{Z}_{+} .
$$

Let

$$
\begin{aligned}
A & =\{(j, k): 0 \leq j<r, k \geq r+1\}, \\
B_{q} & =\{(j, k): 0 \leq j \leq r-1, r q \leq k-(j+1) \leq r(q+1)\} \quad(q \geq 1), \\
B & =\bigcup_{q \geq 1} B_{q} .
\end{aligned}
$$


Clearly, $A \supseteq B$. It follows from (3.6) that

$$
\text { if }(j, k) \in B_{q} \text { then } a(j, k) \geq \alpha_{q+1} / r \text { for } q \geq 1 .
$$

Applying (3.6) and (3.7), we get

$$
\begin{aligned}
\left\|T_{m}(a)\right\|_{\mathcal{M}_{1}} & =\alpha_{0} m_{0}+\alpha_{1} m_{1}+\sum_{(j, k) \in A} a(j, k) m_{k-j} \\
& \geq \alpha_{0} m_{0}+\alpha_{1} m_{1}+\sum_{(j, k) \in B} a(j, k) m_{k-j} \\
& =\alpha_{0} m_{0}+\alpha_{1} m_{1}+\sum_{q \geq 1} \sum_{(j, k) \in B_{q}} a(j, k) m_{k-j} \\
& \geq \alpha_{0} m_{0}+\alpha_{1} m_{1}+r^{-1} \sum_{q \geq 1} \sum_{(j, k) \in B_{q}} \alpha_{q+1} m_{k-j} .
\end{aligned}
$$

Note that, for a fixed $q \geq 1$,

$$
\begin{aligned}
\sum_{(j, k) \in B_{q}} r^{-1} \alpha_{q+1} m_{k-j} & =\sum_{0 \leq j<r} \sum_{k=r q+j}^{r(q+1)+j} r^{-1} \alpha_{q+1} m_{k-j}=\sum_{0 \leq j<r} \sum_{s=r q+1}^{r(q+1)} r^{-1} \alpha_{q+1} m_{s} \\
& =\alpha_{q+1} \sum_{s=r q+1}^{r(q+1)} m_{s} .
\end{aligned}
$$

Thus,

$$
\left\|T_{m}(a)\right\|_{\mathcal{M}_{1}} \geq \alpha_{0} m_{0}+\alpha_{1} m_{1}+\sum_{q \geq 1} \alpha_{q+1} \sum_{s=r q+1}^{(q+1) r} m_{s}
$$

Recall that $a$ depends only on a fixed $r$. Taking in (3.8) the supremum over all eventually zero, non-negative $\left(\alpha_{q}\right)_{q \in \mathbb{Z}_{+}}$with $\sum_{q \in \mathbb{Z}_{+}} \alpha_{q}^{2}=1$, we obtain

$$
\left\|T_{m}(a)\right\|_{\mathcal{M}_{1}} \geq\left(m_{0}^{2}+m_{1}^{2}+\sum_{q \geq 1}\left(\sum_{s=r q+1}^{(q+1) r} m_{s}\right)^{2}\right)^{1 / 2} .
$$

Thus, taking the supremum over $r \geq 1$ and invoking (3.5), we obtain the left hand inequality of (3.1).

Recall that the multiplier $(1 /(s+1))_{s \in \mathbb{Z}_{+}}$corresponds to the classical Hardy inequality $\sum_{s \in \mathbb{Z}_{+}}|\widehat{f}(s)| /(s+1)<\infty$ for $f \in H^{1}(\mathbb{T})$. Thus

Corollary 3.4 ([Sh]). If $a \in \mathcal{U} \mathcal{T} \mathcal{S}_{1}$ then

$$
\sum_{j \geq 0} \sum_{k \geq j} \frac{|a(j, k)|}{k-j+1}<\infty .
$$


REMARK 3.5. Let

$$
\varrho_{2}(m)=\sup _{N \in \mathbb{Z}_{+}}\left((N+1)^{-2} \sum_{s=0}^{N}(s+1)^{2}\left|m_{s}\right|^{2}\right)^{1 / 2},
$$

where $m=\left(m_{s}\right)_{s \in \mathbb{Z}_{+}}$is a scalar sequence. A classical result of Paley, and Hardy and Littlewood (cf. [Pa 1, HL], [Du, Ch. 6, Theorem 6.7]) asserts that $m$ is a Fourier multiplier from $H_{1}$ to $\ell_{2}\left(\mathbb{Z}_{+}\right)$if and only if $\varrho_{2}(m)<\infty$. More generally it follows from [BP], Theorem 3.3 and Proposition 3.1 that

(*) There is $c>0$ such that for every scalar sequence $m$,

$$
\sum_{s \in \mathbb{Z}_{+}}\left|m_{s}\right|^{2}\|\widehat{f}(s)\|_{1}^{2} \leq c^{2} \varrho_{2}(m)^{2}\|f\|_{H_{1}\left(\mathbb{T} ; \mathcal{S}_{1}\right)}^{2}, \quad f \in H_{1}\left(\mathbb{T} ; \mathcal{S}_{1}\right) .
$$

Thus, with the same constant $c$, we have

PRoposition 3.6. For any scalar sequence $m=\left(m_{s}\right)_{s \in \mathbb{Z}_{+}}$one has

$$
\varrho_{2}(m) \leq \sup _{\|a\|_{1} \leq 1}\left(\sum_{s \in \mathbb{Z}_{+}}\left|m_{s}\right|^{2}\left\|d_{s}(a)\right\|_{1}^{2}\right)^{1 / 2} \leq c \varrho_{2}(m)
$$

where $d_{s}(a)$ is defined by (3.2).

Proof. The proof of the right hand inequality of (3.9) is almost the same as the proof of the right hand inequality of (3.1) with Theorem 3.2 replaced by $(*)$. To establish the left hand inequality of (3.9) for fixed $N \in \mathbb{Z}_{+}$define $a^{(N)} \in \mathcal{U} \mathcal{T} \mathcal{S}_{1}$ by

$$
a^{(N)}(j, k)= \begin{cases}(N+1)^{-1} & \text { for } 0 \leq j \leq N \text { and } N+1 \leq k \leq 2 N+1, \\ 0 & \text { otherwise. }\end{cases}
$$

Then $\left\|a^{(N)}\right\|_{1}=1$ and $\left\|d_{s}\left(a^{(N)}\right)\right\|_{1}=\frac{s+1}{N+1}$ for $s=0,1, \ldots, N$. Thus,

$$
(N+1)^{-2} \sum_{s=0}^{N}(s+1)^{2}\left|m_{s}\right|^{2}=\sum_{s=0}^{N}\left\|d_{s}\left(a^{(N)}\right)\right\|_{1}^{2}\left|m_{s}\right|^{2} \leq \sum_{s \in \mathbb{Z}_{+}}\left\|d_{s}\left(a^{(N)}\right)\right\|_{1}^{2}\left|m_{s}\right|^{2} .
$$

Letting $N \rightarrow \infty$, we get

$$
\begin{aligned}
\varrho_{2}(m) & =\sup _{N \in \mathbb{Z}_{+}}(N+1)^{-1}\left(\sum_{s \in \mathbb{Z}_{+}}(s+1)^{2}\left|m_{s}\right|^{2}\right)^{1 / 2} \\
& \leq \sup _{\|a\|_{1}=1}\left(\sum_{s \in \mathbb{Z}_{+}}\left\|d_{s}(a)\right\|^{2}\left|m_{s}\right|^{2}\right)^{1 / 2}
\end{aligned}
$$

which is equivalent to the left hand inequality of (3.9). 
It was observed by Prof. F. Lust-Piquard that $\left\|T_{m}: \mathcal{U T} \mathcal{S}_{1} \rightarrow \mathcal{M}_{2}\right\|=$ $\|m\|_{\infty}$ because $\mathcal{U} \mathcal{T} \mathcal{S}_{1} \subset \mathcal{U} \mathcal{T} \mathcal{S}_{2}=\mathcal{U} \mathcal{T} \mathcal{M}_{2}$. Thus in contrast to the result of Paley and Hardy and Littlewood mentioned above, the norms $\varrho_{2}(m)$ and $\left\|T_{m}: \mathcal{U} \mathcal{T} \mathcal{S}_{1} \rightarrow \mathcal{M}_{2}\right\|$ are not equivalent.

4. Schur multipliers from $B\left(\ell_{2}\right)$ into $\mathcal{M}_{1}$. We describe the space $\mathbb{S}:=\mathbb{S}\left(B\left(\ell_{2}\right), \mathcal{M}_{1}\right)$ of all Schur multipliers from $B\left(\ell_{2}\right)$ into $\mathcal{M}_{1}$. Note that the zero matrix is the only Toeplitz multiplier from $B\left(\ell_{2}\right)$ into $\mathcal{M}_{1}$. To formulate the main result of this section we define the following norms for $a \in \mathcal{M}$ :

$$
\begin{aligned}
\|a\|_{\ell_{p}\left(\ell_{2}\right)}= & \left\|\left(\sum_{k \in \mathbb{Z}_{+}}|a(\cdot, k)|^{2}\right)^{1 / 2}\right\|_{\ell_{p}} \quad(1 \leq p \leq \infty), \\
\beta(a)= & \inf _{a=a^{\prime}+a^{\prime \prime}}\left\{\left\|a^{\prime}\right\|_{\ell_{1}\left(\ell_{2}\right)}+\left\|^{t} a^{\prime \prime}\right\|_{\ell_{1}\left(\ell_{2}\right)}\right\} \\
= & \inf _{a=a^{\prime}+a^{\prime \prime}}\left\{\sum_{j \in \mathbb{Z}_{+}}\left(\sum_{k \in \mathbb{Z}_{+}}\left|a^{\prime}(j, k)\right|^{2}\right)^{1 / 2}\right. \\
& \left.+\sum_{k \in \mathbb{Z}_{+}}\left(\sum_{j \in \mathbb{Z}_{+}}\left|a^{\prime \prime}(j, k)\right|^{2}\right)^{1 / 2}\right\}, \\
\alpha(a)= & \sup _{\varepsilon^{\natural} \equiv 1}\|\varepsilon \diamond a\|_{1} .
\end{aligned}
$$

TheORem 4.1. For every $a \in \mathcal{M}$ we have $\alpha(a)<\infty$ if and only if $\beta(a)<\infty$. Moreover

$$
\left\|S_{a}: B\left(\ell_{2}\right) \rightarrow \mathcal{M}_{1}\right\|=\alpha(a)
$$

and there is an absolute constant $C<\infty$ such that

$$
\alpha(a) \leq \beta(a) \leq C \alpha(a) \quad \forall a \in \mathcal{M} .
$$

The essential part of Theorem 4.1 is the inequality (4.5). It follows from results in $[\mathrm{Lu}]$ and extends to the non-commutative setting Nazarov's [N] generalization of the Kahane-Katznelson-de Leeuw [KKL] theorem on Fourier coefficients. The shortest way to get (4.5) is to use the characterization of multipliers from $c_{0}\left(\mathbb{Z}_{+} \times \mathbb{Z}_{+}\right)$into $\mathcal{S}_{1}[\mathrm{Lu}$, p. 373] and dualize it. However we derive (4.5) from the central statement [Lu, Theorem 2]:

(LP) There is an absolute constant $K>0$ such that if $\phi \in \mathcal{M}$ satisfies

$$
\max \left(\|\phi\|_{\ell_{\infty}\left(\ell_{2}\right)},\|\|^{t} \phi \|_{\ell_{\infty}\left(\ell_{2}\right)}\right) \leq 1,
$$

then there exists a $\psi \in B\left(\ell_{2}\right)$ such that $\|\psi\|_{B\left(\ell_{2}\right)} \leq K$ and $|\phi(j, k)|$ $\leq|\psi(j, k)|$ for $(j, k) \in \mathbb{Z}_{+} \times \mathbb{Z}_{+}$. 
Proof of Theorem 4.1. The trace duality between $\mathcal{S}_{1}$ and $B\left(\ell_{2}\right)$ yields the equality (4.4):

$$
\begin{aligned}
\left\|S_{a}: B\left(\ell_{2}\right) \rightarrow \mathcal{M}_{1}\right\| & =\sup _{\|b\|_{B\left(\ell_{2}\right)}=1} \sum_{(j, k) \in \mathbb{Z}_{+} \times \mathbb{Z}_{+}}|a(j, k) b(j, k)| \\
& =\sup _{\|b\|_{B\left(\ell_{2}\right)}=1} \sup _{\varepsilon^{\natural} \equiv 1} \sum_{(j, k) \in \mathbb{Z}_{+} \times \mathbb{Z}_{+}} \varepsilon(j, k) a(j, k) b(j, k) \\
& \left.=\sup _{\varepsilon^{\natural} \equiv 1\|b\|_{B\left(\ell_{2}\right)=1}} \sup { }^{t}(\varepsilon \diamond a) b\right) \\
& =\sup _{\varepsilon^{\natural} \equiv 1}\left\|^{t}(\varepsilon \diamond a)\right\|_{1}=\sup _{\varepsilon^{\natural} \equiv 1}\|\varepsilon \diamond a\|_{1} .
\end{aligned}
$$

The left hand inequality of (4.5) is easy. Fix $a \in \mathcal{M}$ with $\beta(a)<\infty$. For $\eta>0$ pick $a^{\prime}$ and $a^{\prime \prime}$ so that $a=a^{\prime}+a^{\prime \prime}$ and

$$
\left\|a^{\prime}\right\|_{\ell_{1}\left(\ell_{2}\right)}+\left\|^{t} a^{\prime \prime}\right\|_{\ell_{1}\left(\ell_{2}\right)}<\beta(a)+\eta \text {. }
$$

Let ${ }^{j} a^{\prime}$ denote the $j$ th row of $a^{\prime}$. Since $\alpha\left({ }^{j} a^{\prime}\right)=\left(\sum_{k \in \mathbb{Z}_{+}}\left|a^{\prime}(j, k)\right|^{2}\right)^{1 / 2}$, the triangle inequality for $\alpha$ yields $\alpha\left(a^{\prime}\right) \leq\left\|a^{\prime}\right\|_{\ell_{1}\left(\ell_{2}\right)}$. The same argument applied to the columns of $a^{\prime \prime}$ gives $\alpha\left(a^{\prime \prime}\right) \leq\left\|{ }^{t} a^{\prime \prime}\right\|_{\ell_{1}\left(\ell_{2}\right)}$. Adding these two inequalities together, taking into account that $\alpha(a) \leq \alpha\left(a^{\prime}\right)+\alpha\left(a^{\prime \prime}\right)$ and letting $\eta \rightarrow 0$, we get the left hand inequality of (4.5).

For the right hand inequality of (4.5) we need the following known fact:

Lemma 4.2. Let $\beta^{*}(\phi):=\sup _{\beta(a) \leq 1} \operatorname{Tr}(a \phi)$ for $\phi \in \mathcal{M}$. Then

$$
\beta^{*}(\phi)=\max \left(\|\phi\|_{\ell_{\infty}\left(\ell_{2}\right)},\|\|^{t} \phi \|_{\ell_{\infty}\left(\ell_{2}\right)}\right) .
$$

For the sake of completeness we give the proof of Lemma 4.2 after we finish the proof of Theorem 4.1.

Let us consider the spaces and balls

$$
\begin{aligned}
\left(\mathcal{M}, \beta^{*}\right) & =\left\{a \in \mathcal{M}: \beta^{*}(a)<\infty\right\}, & (\mathcal{M}, \beta) & =\{a \in \mathcal{M}: \beta(a)<\infty\}, \\
B_{\alpha} & =\{a \in \mathcal{M}: \alpha(a) \leq 1\}, & B_{\beta} & =\{a \in \mathcal{M}: \beta(a) \leq 1\} .
\end{aligned}
$$

It follows from Lemma 4.2 that $\left(M, \beta^{*}\right)$ is the dual of $(M, \beta)$ in the trace duality.

Pick $a \in \mathcal{M}$ with $\beta(a) \geq 1$. Since $B_{\beta}$ is a balanced closed (in the norm $\beta$ ) convex set, the separation theorem yields the existence of a functional $\phi$ such that

$$
\begin{aligned}
\operatorname{Tr}(a \phi) & \geq 1, \\
\beta^{*}(\phi) & \leq 1 .
\end{aligned}
$$

(4.8) implies that there exists $\psi$ as in (LP). Hence, there exist $\varepsilon \in \mathcal{M}$ and 
$b \in \mathcal{M}$ such that

$$
\begin{aligned}
& \varepsilon^{\natural} \equiv 1, \quad b(j, k) \geq 1 \quad \text { for }(j, k) \in \mathbb{Z}_{+} \times \mathbb{Z}_{+}, \\
& \psi=\varepsilon \diamond b \diamond \phi \quad \text { with }\|\psi\|_{\infty} \leq K .
\end{aligned}
$$

Define $\sigma \in \mathcal{M}$ by

$$
\sigma(j, k)= \begin{cases}\overline{\varepsilon(j, k)} \operatorname{sign}(\phi(j, k) a(k, j)) \\ \text { for }(j, k) \in \mathbb{Z}_{+} \times \mathbb{Z}_{+} \text {and } \phi(j, k) a(k, j) \neq 0, \\ \text { otherwise. }\end{cases}
$$

Clearly $\sigma^{\natural} \equiv 1$. Thus, invoking (LP), (4.7) and (4.9), we get

$$
\begin{aligned}
|\operatorname{Tr}((\psi \diamond \sigma) a)|=\left|\sum_{(j, k) \in \mathbb{Z}_{+} \times \mathbb{Z}_{+}} \psi(j, k) \sigma(j, k) a(k, j)\right| \\
=\left|\sum_{(j, k) \in \mathbb{Z}_{+} \times \mathbb{Z}_{+}} \varepsilon(j, k) b(j, k) \phi(j, k) \overline{\varepsilon(j, k)} \operatorname{sign}(\phi(j, k) a(k, j)) a(k, j)\right| \\
=\sum_{(j, k) \in \mathbb{Z}_{+} \times \mathbb{Z}_{+}} b(j, k)|\phi(j, k) a(k, j)| \geq \sum_{(j, k) \in \mathbb{Z}_{+} \times \mathbb{Z}_{+}}|\phi(j, k) a(k, j)| \\
\quad \geq\left|\sum_{(j, k) \in \mathbb{Z}_{+} \times \mathbb{Z}_{+}} \phi(j, k) a(k, j)\right|=|\operatorname{Tr}(\phi a)| \geq 1 .
\end{aligned}
$$

Invoking (4.3) and the trace duality between $B\left(\ell_{2}\right)$ and $\mathcal{S}_{1}$, by (4.9), we get

$$
1 \leq|\operatorname{Tr}((\psi \diamond \sigma) a)|=\left|\operatorname{Tr}\left(\psi \cdot\left({ }^{t} \sigma \diamond a\right)\right)\right| \leq\|\psi\|_{B\left(\ell_{2}\right)}\left\|^{t} \sigma \diamond a\right\|_{1} \leq K \alpha(a) .
$$

Hence, we have shown that $\beta(a) \geq 1$ yields $\alpha(a) \geq K^{-1}$, which implies the right hand inequality of (4.5) for $a \in \mathcal{M}$ with $C=K$.

Proof of Lemma 4.2. Put

$$
\widetilde{\beta}(b, c)=\|b\|_{\ell_{1}\left(\ell_{2}\right)}+\left\|{ }^{t} c\right\|_{\ell_{1}\left(\ell_{2}\right)} \quad((b, c) \in \mathcal{M} \oplus \mathcal{M}) .
$$

Consider the space

$$
E=\{(b, c) \in \mathcal{M} \oplus \mathcal{M}: \widetilde{\beta}(b, c)<\infty\} .
$$

equipped with the norm $\widetilde{\beta}$.

Define the map $q: E \rightarrow \mathcal{M}$ by $q(b, c)=b+c$. Obviously $\operatorname{ker} q=$ $\{(b, c): b=-c\}$. A moment's reflection shows that $q$ is a contractive surjection from $E$ onto $(\mathcal{M}, \beta)$, hence the Banach space $(\mathcal{M}, \beta)$ can be identified with the quotient $E / \operatorname{ker} q$. Thus the dual space of $(\mathcal{M}, \beta)$ can be identified with the annihilator of $\operatorname{ker} q$ in the space

$$
E^{*}=\left\{(\phi, \psi) \in \mathcal{M} \oplus \mathcal{M}:(\widetilde{\beta})^{*}<\infty\right\} .
$$

Clearly $(\widetilde{\beta})^{*}(\phi, \psi)=\max \left(\|\phi\|_{\ell_{\infty}\left(\ell_{2}\right)},\left\|{ }^{t} \psi\right\|_{\ell_{\infty}\left(\ell_{2}\right)}\right)$. The annihilator of ker $q$ is $\left\{(\phi, \psi) \in E^{*}: \phi=\psi\right\}$. Indeed, the duality is given by $(\phi, \psi)(b, c)=$ $\operatorname{Tr}(a \phi)+\operatorname{Tr}(b \psi)$. Putting $b=-c$, we obtain $\phi=\psi$. This yields (4.6). 
5. Schur multipliers from $\mathcal{U T} B\left(\ell_{2}\right)$ into $\mathcal{M}_{1}$. The main result of this section, Theorem 5.1, is due to G. Pisier and is published here with his permission. It can be stated as the formula $\mathbb{S}\left(\mathcal{U T} B\left(\ell_{2}\right), \mathcal{M}_{1}\right)=\mathcal{U} \mathcal{T} \mathbb{S}$. Recall that $\mathbb{S}=\mathbb{S}\left(B\left(\ell_{2}\right), \mathcal{M}_{1}\right)$ stands for the space of Schur multipliers from $B\left(\ell_{2}\right)$ into $\mathcal{M}_{1}$ characterized in Theorem 4.1. Precisely, we shall prove

THEOREM 5.1. An element $a \in \mathcal{U} \mathcal{T} \mathcal{M}$ induces a Schur multiplier $S_{a}$ : $\mathcal{U T} B\left(\ell_{2}\right) \rightarrow \mathcal{M}_{1}$ if and only if $a \in \mathcal{U T} \mathbb{S}$. Equivalently, there is an absolute constant $K_{*}>0$ such that

$$
K_{*}^{-1} \alpha(a) \leq \gamma(a) \leq \alpha(a), \quad \text { where } \gamma(a)=\left\|S_{a}: \mathcal{U T} B\left(\ell_{2}\right) \rightarrow \mathcal{M}_{1}\right\| .
$$

Note that in this section we use $S_{a}$ to denote Schur multipliers induced by elements $a \in \mathcal{U T} \mathcal{M}$ or $a \in \mathcal{M}$ acting on the space $\mathcal{U T} B\left(\ell_{2}\right)$, while Schur multipliers induced by elements $a \in \mathcal{M}$ acting on $B\left(\ell_{2}\right)$ are denoted by $\widetilde{S}_{a}$. We begin with

Lemma 5.2. $\mathcal{U} \mathcal{T} \mathcal{M}_{0}$ is dense in $\mathcal{U T S}$ in the norm $\gamma(\cdot)$.

Outline of proof. Let $e_{j, k}$ denote the unit $(j, k)$-entry matrix. Let $\mathcal{U} \mathcal{T} \mathbb{S}_{0}$ denote the closure of $\mathcal{U} \mathcal{T} \mathcal{M}_{0}$ in the norm $\gamma(\cdot)$. All infinite sums of matrices are regarded in entrywise convergence. Fix $b \in \mathcal{U} \mathcal{T} \mathbb{S}$. Note that

(i) $\gamma\left(\sum_{k=m}^{\infty} b(j, k) e_{j, k}\right)=\left(\sum_{k=m}^{\infty}|b(j, k)|^{2}\right)^{1 / 2}$ for $0 \leq j \leq m<\infty$.

(ii) Let $b_{s}=\sum_{j=0}^{s} \sum_{k=j}^{\infty} b(j, k) e_{j, k}$ for $s \in \mathbb{Z}_{+}$. Then $b_{s} \in \mathcal{U} \mathcal{T} \mathbb{S}_{0}$ and $\lim _{s} \gamma\left(b_{s}\right)=\gamma(b)$.

(iii) $\lim _{s}\left\|\left(b-b_{s}\right) \diamond a\right\|_{\mathcal{M}_{1}}=0$ for every $a \in B\left(\ell_{2}\right)$.

Assume to the contrary that there exists $b \in \mathcal{U} \mathcal{T} \mathbb{S} \backslash \mathcal{U} \mathcal{T} \mathbb{S}_{0}$. Then there exists $\delta>0$ such that $\gamma\left(b-b^{\prime}\right)>\delta$ for every $b^{\prime} \in \mathcal{U} \mathcal{T} \mathbb{S}_{0}$. This allows constructing inductively an increasing sequence $\left(s_{\nu}\right)_{\nu \in \mathbb{Z}_{+}}$of indices with $n_{0}=0$ and a sequence $\left(a_{\nu}\right)_{\nu \in \mathbb{Z}_{+}}$of matrices in $\mathcal{U} \mathcal{T} \mathcal{M}_{0}$ such that

$$
\begin{gathered}
\left\|a_{\nu}\right\|_{B\left(\ell_{2}\right)}=1, \quad \operatorname{supp} a_{\nu} \subset\left\{(j, k) \in \mathbb{Z}_{+} \times \mathbb{Z}_{+}: s_{\nu} \leq j \leq k<s_{\nu+1}\right\}, \\
\left\|\left(b-b_{s_{\nu+1}}\right) \diamond a_{\nu}\right\|_{\mathcal{M}_{1}}<2^{-(\nu+2)} \delta, \\
\gamma\left(b-b_{s_{\nu}}\right)>\left\|\left(b-b_{s_{\nu}}\right) \diamond a_{\nu}\right\|_{\mathcal{M}_{1}}=\left\|\left(b_{s_{\nu+1}}-b_{s_{\nu}}\right) \diamond a_{\nu}\right\|_{\mathcal{M}_{1}}>\left(1-2^{-(\nu+2)}\right) \delta .
\end{gathered}
$$

Put $a=\sum_{\nu=0}^{\infty} a_{\nu}$. Since the supports of the $a_{\nu}$ 's are contained in the consecutive rectangles whose projections onto the $j$-axis and $k$-axis are disjoint, $\|a\|_{B\left(\ell_{2}\right)}=\sup _{\nu}\left\|a_{\nu}\right\|_{B\left(\ell_{2}\right)}=1$. We have $b=b_{s_{0}}+\sum_{\nu=0}^{\infty}\left(b_{s_{\nu+1}}-b_{s_{\nu}}\right)$. Hence

$$
\begin{aligned}
\|b \diamond a\|_{\mathcal{M}_{1}} & =\left\|b_{s_{0}} \diamond a\right\|_{\mathcal{M}_{1}}+\sum_{\nu=0}^{\infty}\left\|\left(b_{s_{\nu+1}}-b_{s_{\nu}}\right) \diamond a\right\|_{\mathcal{M}_{1}} \\
& =\left\|b_{s_{0}} \diamond a_{0}\right\|_{\mathcal{M}_{1}}+\sum_{\nu=0}^{\infty}\left\|\left(b_{s_{\nu+1}}-b_{s_{\nu}}\right) \diamond a_{\nu+1}\right\|_{\mathcal{M}_{1}}>\sum_{\nu=0}^{\infty} \delta / 2=\infty .
\end{aligned}
$$

A contradiction. 
Since $\widetilde{S}_{a}$ extends $S_{a}$, the right hand inequality of (5.1) is obvious. To prove the left hand inequality we need more notation. Let $|x|_{\mathrm{s}}=\left(x^{*} x+x x^{*}\right)^{1 / 2}$ for $x \in B\left(\ell_{2}\right)$. Call a matrix $d$ a diagonal state provided that $d(j, k)=0$ for $(j, k) \in \mathbb{Z}_{+} \times \mathbb{Z}_{+}$with $j \neq k$, and $d_{j}:=d(j, j)>0$ for $j \in \mathbb{Z}_{+}$, and $\operatorname{Tr} d=\sum_{j \in \mathbb{Z}_{+}} d_{j}=1$. Our proof essentially uses the concept of $p$ - $C^{*}$ summing operator introduced by G. Pisier [Pi 1, M, PiX, p. 1488]. For our purpose, in the case of Schur upper triangular multipliers, it can be defined as follows:

Definition 5.3. Let $1 \leq p \leq \infty$ and let $a \in \mathcal{U} \mathcal{T} \mathcal{M}_{0}$. The multiplier $S_{a}: \mathcal{U T} B\left(\ell_{2}\right) \rightarrow \mathcal{M}_{1}$ is $p-C^{*}$-summing provided that there exist a diagonal state $d$ and a non-negative constant $C_{p}(a, d)$ such that

$$
\|a \diamond x\|_{\mathcal{M}_{1}} \leq C_{p}(a, d) \operatorname{Tr}\left(d|x|_{\mathrm{S}}^{p}\right)^{1 / p} \quad \text { for } x \in \mathcal{U} \mathcal{T} B\left(\ell_{2}\right) .
$$

Define the $p$ - $C^{*}$-summing constant by $C_{p}(a)=\inf \left\{C_{p}(a, d): d\right.$ satisfies (5.2)\}. The symbol $\widetilde{S}_{a}$ stands for the multiplier from $B\left(\ell_{2}\right)$ into $\mathcal{M}_{1}$. The multiplier $\widetilde{S}_{a}: B\left(\ell_{2}\right) \rightarrow \mathcal{M}_{1}$ is $p$ - $C^{*}$-summing provided it satisfies (5.2) for $x \in B\left(\ell_{2}\right)$; the $p$ - $C^{*}$-summing constant of $\widetilde{S}_{a}$ is denoted by $\widetilde{C}_{p}(a)$.

REMARK 5.4. (i) $p$ - $C^{*}$-summing operators can be defined in terms of domination by states which play the role of Pietsch measures (cf. [PiX, pp. 1488-1489]). The definition extends to subspaces of $C^{*}$-algebras. Prof. G. Pisier observed (oral communication) that for Schur multipliers from $\mathcal{U T} B\left(\ell_{2}\right)$ and $B\left(\ell_{2}\right)$ general states can be replaced by diagonal ones. This justifies Definition 5.3.

(ii) If $a \in \mathcal{U} \mathcal{T} \mathcal{M}_{0}$ then $C_{p}(a, d)<\infty$ for every diagonal state $d$ (enough to check for matrices with only one entry different from zero).

(iii) Let $1 \leq p_{1} \leq p_{2}<\infty$. If (5.2) is satisfied for some diagonal state $d$ for $p_{1}$ with $C_{p_{1}}(a, d)$ then it is satisfied for $p_{2}$ with the same constant $C_{p_{1}}(a, d)$. Hence $C_{p_{2}}(a) \leq C_{p_{1}}(a)$.

Proposition 5.5. Let $\beta(\cdot)$ be defined by (4.2). If $S_{a}: \mathcal{U T} B\left(\ell_{2}\right) \rightarrow \mathcal{M}_{1}$ is $2-C^{*}$-summing then $\beta(a) \leq 2 \sqrt{2} C_{2}(a)$.

Proof. By Lemma 5.2, it is enough to establish the assertion for $a \in$ $\mathcal{U} \mathcal{T} \mathcal{M}_{0}$. Fix a diagonal state $d$. Put $\Lambda=\left\{(j, k) \in \mathbb{Z}_{+} \times \mathbb{Z}_{+}: j \leq k\right\}, \Lambda^{\prime}=$ $\left\{(j, k) \in \Lambda: d_{j} \geq d_{k}\right\}, \Lambda^{\prime \prime}=\Lambda \backslash \Lambda^{\prime}$. Let $a^{\prime}=1_{\Lambda^{\prime}} \diamond a$ and $a^{\prime \prime}=1_{\Lambda^{\prime \prime}} \diamond a$.

It is sufficient to show that

$$
\left\|a^{\prime}\right\|_{\ell_{1}\left(\ell_{2}\right)} \leq \sqrt{2} C_{2}(a) \text { and }\left\|^{t} a^{\prime \prime}\right\|_{\ell_{1}\left(\ell_{2}\right)} \leq \sqrt{2} C_{2}(a)
$$

We shall prove only the first inequality, since the proof of the second is similar. To this end, we first show that

$$
\left\|d^{-1 / 2} a^{\prime} \diamond x\right\|_{\mathcal{M}_{1}} \leq \sqrt{2} C_{2}(a)\|x\|_{2} \quad\left(x \in \mathcal{U} \mathcal{T} \mathcal{M}_{0}\right)
$$


The linearity and commutativity of the trace imply

$$
\left\|S_{a} x\right\|_{\mathcal{M}_{1}} \leq C_{2}(a, d)\left(\operatorname{Tr}\left(d|x|_{\mathrm{s}}^{2}\right)\right)^{1 / 2}=C_{2}(a, d)\left(\operatorname{Tr}\left(d x^{*} x+x x^{*} d\right)\right)^{1 / 2} .
$$

Thus

$$
\begin{aligned}
\left\|S_{a^{\prime}} x\right\|_{\mathcal{M}_{1}} & =\left\|S_{a}\left(1_{\Lambda^{\prime}} \diamond x\right)\right\|_{\mathcal{M}_{1}} \\
& \leq C_{2}(a, d)\left(\operatorname{Tr}\left(\sum_{(j, k) \in \Lambda^{\prime}} d_{j}|x(j, k)|^{2}+|x(j, k)|^{2} d_{k}\right)\right)^{1 / 2} \\
& \leq C_{2}(a, d)\left(\operatorname{Tr}\left(\sum_{(j, k) \in \Lambda^{\prime}} 2 d_{j}|x(j, k)|^{2}\right)\right)^{1 / 2} \quad\left(\text { by definition of } \Lambda^{\prime}\right) \\
& =C_{2}(a, d) \sqrt{2}\left\|d^{1 / 2} x\right\|_{2} .
\end{aligned}
$$

Replacing $x$ by $d^{-1 / 2} x$, we obtain (5.4). On the other hand, remembering that $\sum_{j \in \mathbb{Z}_{+}} d_{j}=1$ and applying the Schwarz inequality, we get

$$
\begin{aligned}
\left\|a^{\prime}\right\|_{\ell_{1}\left(\ell_{2}\right)} & =\sum_{j \in \mathbb{Z}_{+}}\left(\sum_{k \in \mathbb{Z}_{+}}\left|a^{\prime}(j, k)\right|^{2}\right)^{1 / 2} \\
& =\sum_{j \in \mathbb{Z}_{+}} d_{j}^{1 / 2}\left(\sum_{k \in \mathbb{Z}_{+}} d_{j}^{-1}\left|a^{\prime}(j, k)\right|^{2}\right)^{1 / 2} \\
& \leq\left(\sum_{j \in \mathbb{Z}_{+}} d_{j}\right)^{1 / 2}\left(\sum_{(j, k) \in \Lambda} d_{j}^{-1}\left|a^{\prime}(j, k)\right|^{2}\right)^{1 / 2} \\
& =\left(\sum_{(j, k) \in \Lambda} d_{j}^{-1}\left|a^{\prime}(j, k)\right|^{2}\right)^{1 / 2} .
\end{aligned}
$$

Since $\sum_{(j, k) \in \Lambda}\left|d_{j}^{-1 / 2} a^{\prime}(j, k) x(j, k)\right|=\left\|d^{-1 / 2} a^{\prime} \diamond x\right\|_{\mathcal{M}_{1}}$, taking the supremum in (5.4) over all $x \in \mathcal{U} \mathcal{T} \mathcal{M}_{0}$ with $\sum_{(j, k) \in \Lambda}|x(j, k)|^{2}=1$ we get

$$
\left(\sum_{(j, k) \in \Lambda} d_{j}^{-1}\left|a^{\prime}(j, k)\right|^{2}\right)^{1 / 2} \leq C_{2}(a, d) \sqrt{2} .
$$

Combining the latter inequality with (5.5) we obtain

$$
\left\|a^{\prime}\right\|_{\ell_{1}\left(\ell_{2}\right)} \leq \sqrt{2} C_{2}(a, d) \text {. }
$$

Taking in (5.6) the infimum over all diagonal states $d$ we obtain the first inequality in (5.3).

The proof of the next proposition heavily depends on interpolation theory.

Proposition 5.6. There exists $L_{*}>0$ such that, for every $a \in \mathcal{U} \mathcal{T} \mathcal{M}_{0}$,

$$
\widetilde{C}_{4}(a) \leq L_{*} \sqrt{C_{2}(a) \gamma(a)} .
$$


Proof. Fix a diagonal state $d$. Since $a \in \mathcal{U} \mathcal{T} \mathcal{M}_{0}$, we may assume that $C_{2}(a, d)<\infty$. Define $a^{\prime}$ and $a^{\prime \prime}$ as in the proof of Proposition 5.5. We shall work first with $a^{\prime}$. Assume that $x \in \mathcal{U} \mathcal{T} \mathcal{M}_{0}$. From the definition of $\gamma(a)$, noting that $\left\|a^{\prime} \diamond x\right\|_{\mathcal{M}_{1}} \leq\|a \diamond x\|_{\mathcal{M}_{1}}$, and applying (5.4), we have

$$
\begin{aligned}
\left\|a^{\prime} \diamond x\right\|_{\mathcal{M}_{1}} & \leq \gamma(a)\|x\|_{B\left(\ell_{2}\right)}, \\
\left\|d^{-1 / 2} a^{\prime} \diamond x\right\|_{\mathcal{M}_{1}} & \leq \sqrt{2} C_{2}(a, d)\|x\|_{2} .
\end{aligned}
$$

Since $\left|d_{j}^{(-1 / 2) i y}\right|=1$ for every real $y$ and for every $j \in \mathbb{Z}_{+}$, it follows from (5.8) and (5.9) that

$$
\begin{aligned}
& \left\|d^{(-1 / 2) z} a^{\prime} \diamond x\right\|_{\mathcal{M}_{1}} \leq \gamma(a)\|x\|_{B\left(\ell_{2}\right)} \quad \text { for } \Re z=0, \\
& \left\|d^{-(1 / 2) z} a^{\prime} \diamond x\right\|_{\mathcal{M}_{1}} \leq \sqrt{2} C_{2}(a, d)\|x\|_{2} \quad \text { for } \Re z=1 .
\end{aligned}
$$

It follows from a result of Pisier [Pi 2] (see also Remark (i) after Lemma 8.5 in $[\mathrm{PiX}])$ that the couple $\left(\mathcal{U} \mathcal{T} B\left(\ell_{2}\right), \mathcal{U} \mathcal{T} \mathcal{S}_{2}\right)$ is K-closed in the couple $\left(B\left(\ell_{2}\right), \mathcal{S}_{2}\right)$. Now, applying the interpolation result of [GK, Ch. III, §5], we obtain, for $\theta=1 / 2$,

$$
\left\|d^{(-1 / 2) \cdot(1 / 2)} a^{\prime} \diamond x\right\|_{\mathcal{M}_{1}} \leq L \sqrt{\gamma(a) C_{2}(a, d)}\|x\|_{4} \quad \text { for } x \in \mathcal{U} \mathcal{T} \mathcal{M}_{0},
$$

where the constant $L$ depends only on the couple.

Let $P: \mathcal{M}_{0} \rightarrow \mathcal{M}_{0}$ be the main triangular projection, namely $P y=1_{\Lambda} \diamond y$ for $y \in \mathcal{M}_{0}$. Recall that (cf. e.g. [GK, Ch. III, §6, Theorem 6.2])

$$
\text { there is } K_{4}>0 \text { such that }
$$

$$
\operatorname{Tr}\left(|P y|^{4}\right) \leq K_{4}^{4} \operatorname{Tr}\left(|y|^{4}\right) \quad \text { for every } y \in \mathcal{M}_{0} .
$$

Combining (5.10) and (5.11) yields

$$
\begin{aligned}
\left\|d^{-1 / 4} a^{\prime} \diamond y\right\|_{\mathcal{M}_{1}} & =\left\|d^{-1 / 4} \diamond P y\right\|_{\mathcal{M}_{1}} \leq L \sqrt{\gamma(a) C_{2}(a, d)}\|P y\|_{4} \\
& \leq L K_{4} \sqrt{\gamma(a) C_{2}(a, d)}\|y\|_{4}, \quad \text { for } y \in \mathcal{M}_{0} .
\end{aligned}
$$

Substituting $d^{1 / 4} y$ in place of $y$, we obtain

$$
\left\|a^{\prime} \diamond y\right\|_{\mathcal{M}_{1}} \leq L K_{4} \sqrt{\gamma(a) \cdot C_{2}(a, d)}\left(\operatorname{Tr}\left(\left|d^{1 / 4} y\right|^{4}\right)^{1 / 4} \quad \text { for } y \in \mathcal{M}_{0} .\right.
$$

Using the commutativity of the trace several times, the fact that $\operatorname{Tr}(A \cdot B) \geq 0$ whenever $A$ and $B$ are positive matrices, and $|y|_{\mathrm{s}}^{2}-\left|y^{*}\right|^{2} \geq 0$, we get

$$
\begin{aligned}
\operatorname{Tr}\left(\left|d^{1 / 4} y\right|^{4}\right) & =\operatorname{Tr}\left(y^{*} d^{1 / 2} y y^{*} d^{1 / 2} y\right)=\operatorname{Tr}\left(d^{1 / 2}\left|y^{*}\right|^{2} d^{1 / 2}\left|y^{*}\right|^{2}\right) \\
& \leq \operatorname{Tr}\left(d^{1 / 2}\left|y^{*}\right|^{2} d^{1 / 2}|y|_{\mathrm{s}}^{2}\right) \leq \operatorname{Tr}\left(d^{1 / 2}|y|_{\mathrm{s}}^{2} d^{1 / 2}|y|_{\mathrm{s}}^{2}\right) \\
& =\left\||y|_{\mathrm{s}} d^{1 / 2}|y|_{\mathrm{s}}\right\|_{2}^{2} .
\end{aligned}
$$

Notice that

$$
\left\||y|_{\mathrm{s}} d^{1 / 2}|y|_{\mathrm{s}}\right\|_{2}^{2} \leq\left\|d^{1 / 2}|y|_{\mathrm{s}}^{2}\right\|_{2}^{2}=\operatorname{Tr}\left(|y|_{\mathrm{s}} d|y|_{\mathrm{s}}^{2}\right)=\operatorname{Tr}\left(d|y|_{\mathrm{s}}^{4}\right) .
$$

In the latter inequality we use the fact that if $A$ and $Z$ are positive hermitian matrices in $\mathcal{M}_{0}$ then $\|A Z A\|_{2} \leq\left\|Z A^{2}\right\|_{2}$. This is easy to see if we choose an 
orthonormal basis so that $A$ is a diagonal matrix. Thus

$$
\left\|a^{\prime} \diamond y\right\|_{\mathcal{M}_{1}} \leq L K_{4} \sqrt{\gamma(a) C_{2}(a, d)}\left(\operatorname{Tr}\left(d|y|_{\mathrm{s}}^{4}\right)\right)^{1 / 4} \quad \text { for } y \in \mathcal{M}_{0} .
$$

Similarly, we obtain

$$
\left\|a^{\prime \prime} \diamond y\right\|_{\mathcal{M}_{1}} \leq L K_{4} \sqrt{\gamma(a) C_{2}(a, d)}\left(\operatorname{Tr}\left(d|y|_{\mathrm{s}}^{4}\right)\right)^{1 / 4} \quad \text { for } y \in \mathcal{M}_{0}
$$

Hence, remembering that $a=a^{\prime}+a^{\prime \prime}$ and using the triangle inequality, we infer that

$$
\|a \diamond y\|_{\mathcal{M}_{1}} \leq 2 L K_{4} \sqrt{\gamma(a) C_{2}(a, d)}\left(\operatorname{Tr}\left(d|y|_{\mathrm{s}}^{4}\right)\right)^{1 / 4} \quad \text { for } y \in \mathcal{M}_{0} .
$$

According to Definition 5.3, taking the infimum over all diagonal states, we obtain (5.7).

Proof of Theorem 5.1. We have proved in the proposition above that there exists a constant $L_{*}>0$ such that

$$
\widetilde{C}_{4}(a) \leq L_{*} \sqrt{C_{2}(a) \gamma(a)} \quad \text { for } a \in \mathcal{U} \mathcal{T} \mathcal{M}_{0} .
$$

Now we use an observation of Pisier (cf. [M, Proposition 3]) that every 4- $C^{*}$-summing operator from $B\left(\ell_{2}\right)$ into a Banach space of cotype 2 (in particular, into $\left.\mathcal{M}_{1}=\ell_{1}\left(\mathbb{Z}_{+} \times \mathbb{Z}_{+}\right)\right)$is $2-C^{*}$-summing. Hence, there is a constant $\widetilde{K}>0$ independent of $a$ such that $\widetilde{C}_{2}(a) \leq \widetilde{K} \widetilde{C}_{4}(a)$. Since $\widetilde{S}_{a}$ extends $S_{a}$, we have $C_{2}(a) \leq \widetilde{C}_{2}(a)$. Thus,

$$
C_{2}(a) \leq L_{*} \widetilde{K} \sqrt{C_{2}(a) \gamma(a)} \quad \text { for } a \in \mathcal{U} \mathcal{T} \mathcal{M}_{0},
$$

or, equivalently,

$$
C_{2}(a) \leq L_{*}^{2} \widetilde{K}^{2} \gamma(a) \quad \text { for } a \in \mathcal{U} \mathcal{T} \mathcal{M}_{0}
$$

Combining (5.13) with the assertion of Proposition 5.5, we find that there exists a constant $K_{*}>0$ such that

$$
\beta(a) \leq K_{*} \gamma(a) \quad \text { for } a \in \mathcal{U} \mathcal{T} \mathcal{M}_{0},
$$

and combining this inequality with the left hand inequality of (4.5), we obtain the left hand inequality of (5.1).

REMARK 5.7. Theorem 5.1 can be regarded as a non-commutative analogue of Paley's theorem [Pa 2] which asserts that the space of Fourier multipliers from $H_{\infty}$ (resp. from the disc algebra) into $\ell_{1}\left(\mathbb{Z}_{+}\right)$coincides with $\ell_{2}\left(\mathbb{Z}_{+}\right)$. Theorem 4.1 corresponds to the well known fact that the space of Fourier multipliers from $L_{\infty}(\mathbb{T})$ (resp. from $C(\mathbb{T})$ ) into $\ell_{1}(\mathbb{Z})$ coincides with $\ell_{2}(\mathbb{Z})$. Thus, the Fourier multipliers from $H_{\infty}$ (resp. the disc algebra) into $\ell_{1}\left(\mathbb{Z}_{+}\right)$form a complemented subspace in the space of Fourier multipliers from $L_{\infty}(\mathbb{T})$ (resp. $C(\mathbb{T})$ ) into $\ell_{1}(\mathbb{Z})$ although $H_{\infty}$ is not complemented in $L_{\infty}$ (resp. the disc algebra is not complemented in $C(\mathbb{T})$ ). Similarly, $\mathbb{S}\left(\mathcal{U} \mathcal{T} B\left(\ell_{2}\right), \mathcal{M}_{1}\right)=\mathcal{U} \mathcal{T} \mathbb{S}$ is complemented in $\mathbb{S}=\mathbb{S}\left(B\left(\ell_{2}\right), \mathcal{M}_{1}\right)$, although $\mathcal{U} \mathcal{T} B\left(\ell_{2}\right)$ is not complemented in $B\left(\ell_{2}\right)$. 
6. Hankel matrices and Mazur's problems. In this section we discuss some properties of Hankel matrices related to the problems of Stanisław Mazur (cf. [Scb, Problems 8 and 88]). The problems have been solved independently by P. P. B. Eggermont and Y. J. Leung [EL] and by S. Kwapień and the first named author of the present paper $[\mathrm{KwP}]$. We discuss some qualitative improvement of the solutions. We follow the approach of $[\mathrm{KwP}]$.

Definition 6.1. An $a \in \mathcal{M}$ is said to be a Hankel matrix (notation $a \in \mathcal{H}$ ) provided that

$$
a(j, k)=a(0, j+k) \quad\left((j, k) \in \mathbb{Z}_{+} \times \mathbb{Z}_{+}\right) .
$$

Let

$$
\mathcal{H}_{n}=\mathcal{H} \cap M_{n}=\{a \in \mathcal{H}: a(j, k)=0 \text { for } \max (j, k)>n\} \quad(n=0,1, \ldots) .
$$

Note that

$$
\|a\|_{\mathcal{M}_{1}}=\sum_{k=0}^{\infty}(k+1)|a(0, k)| \quad(a \in \mathcal{H}) .
$$

For $a \in \mathcal{H}$ the series $\phi \sim \sum_{k \geq 0} a^{(k)} \exp (i k t)$, where $a^{(k)}=a(0, k)$ for $k \in \mathbb{Z}_{+}$, is called the symbol of $a$. Conversely, given a series $\phi$ we denote by $a_{[\phi]}$ the corresponding Hankel matrix whose symbol is $\phi$.

Let

$$
\|a\|_{\ell_{1} \hat{\hat{\theta}} \ell_{1}}=\sup \sum_{j \in \mathbb{Z}_{+}}\left|\sum_{k \in \mathbb{Z}_{+}} a(j, k) \sigma_{k}\right| \quad(a \in \mathcal{M}),
$$

the supremum extending over all scalar sequences $\left(\sigma_{k}\right)_{k \in \mathbb{Z}_{+}}$with $\left|\sigma_{k}\right| \leq 1$;

$$
\ell_{1} \hat{\hat{\otimes}} \ell_{1}=\left\{a \in \mathcal{M}:\|a\|_{\ell_{1} \hat{\hat{\otimes}} \ell_{1}}<\infty\right\}
$$

Let

$$
h_{n}=\sup _{a \in \mathcal{H}_{n} ;\|a\|_{\ell_{1} \hat{\hat{\otimes}} \ell_{1}}=1}\|a\|_{\mathcal{M}_{1}} \quad(n=0,1, \ldots) .
$$

Note that the quantities $\|a\|_{\ell_{1} \hat{\hat{\otimes}} \ell_{1}}$ and $h_{n}$ depend on the field of scalars.

S. Mazur asked (cf. [Scb, Problem 88]) whether $a \in \mathcal{H} \cap \ell_{1} \hat{\hat{\otimes}} \ell_{1}$ implies $\|a\|_{\mathcal{M}_{1}}<\infty$.

The negative answer is an immediate consequence of the next proposition combined with the Banach-Steinhaus principle.

Proposition 6.2. There is $C_{*}>0$ such that

$$
h_{n} \geq C_{*} \sqrt{n+1} \quad(n=0,1, \ldots) .
$$

In what follows, $\langle\cdot, \cdot\rangle$ denotes the usual scalar product in $L^{2}(-\pi, \pi)$,

$$
\langle\phi, \psi\rangle=(2 \pi)^{-1} \int_{-\pi}^{\pi} \phi(t) \overline{\psi(t)} d t
$$


Lemma 6.3. Let $\phi(t)=\sum_{k=0}^{n} a^{(k)} \exp (i k t)$ be an analytic trigonometric polynomial of degree $n$. Then

$$
\left\|a_{[\phi]}\right\|_{\ell_{1} \hat{\hat{\otimes}} \ell_{1}} \leq(n+1) \sup _{|t| \leq \pi}|\phi(t)| .
$$

Proof. Pick $(z(k))_{k=0}^{n}$ with $|z(k)| \leq 1$ for $k=0,1, \ldots$ so that

$$
\left\|a_{[\phi]}\right\|_{\ell_{1} \hat{\hat{\otimes}} \ell_{1}}=\sum_{j=0}^{n}\left|\sum_{k=0}^{n-j} a(0, j+k) z(k)\right| .
$$

Define trigonometric polynomials

$$
f(t)=\sum_{k=0}^{n} z(k) \exp (-i k t), \quad g(t)=\sum_{j=0}^{n} \bar{w}_{j} \exp (i j t),
$$

where

$$
w_{j}=\operatorname{sign} \sum_{k=0}^{n-j} z(k) a_{[\phi]}(0, j+k) \quad(j=0,1, \ldots, n) .
$$

Clearly $\phi f=\sum_{j=-n}^{n} d_{j} \exp (i j t)$, where in particular

$$
d_{j}=\sum_{k=0}^{n-j} a_{[\phi]}(0, j+k) z(k) \quad(j=0,1, \ldots, n) .
$$

Thus

$$
\langle\phi f, g\rangle=\sum_{j=0}^{n} d_{j} w_{j}=\sum_{j=0}^{n}\left|\sum_{k=0}^{n-j} a_{[\phi]}(0, j+k) z(k)\right|=\left\|a_{[\phi]}\right\|_{\ell_{1} \hat{\otimes} \ell_{1}} .
$$

On the other hand, using the Cauchy-Schwarz inequality, and taking into account that $\langle f, f\rangle \leq n+1$ and $\langle g, g\rangle \leq n+1$ (because $\operatorname{deg} f<n+1, \operatorname{deg} g<$ $n+1,|z(k)| \leq 1,\left|w_{j}\right| \leq 1$ for $\left.j, k=0,1, \ldots, n\right)$ we obtain

$$
\begin{aligned}
|\langle\phi f, g\rangle| & \leq\langle\phi f, \phi f\rangle^{1 / 2} \cdot\langle g, g\rangle^{1 / 2} \leq \sup _{|t| \leq \pi}|\phi(t)|\langle f, f\rangle^{1 / 2}\langle g, g\rangle^{1 / 2} \\
& \leq(n+1) \sup _{|t| \leq \pi}|\phi(t)| . \text { - }
\end{aligned}
$$

Proof of Proposition 6.2. Let $\left(p_{n}\right)_{n=0}^{\infty}$ be a sequence of analytic trigonometric polynomials such that

(i) $\operatorname{deg} p_{n}=n(n=0,1, \ldots)$;

(ii) the absolute values of the coefficients with indices $\leq n$ of $p_{n}$ are equal to 1 ;

(iii) there is a constant $C>0$ such that

$$
\sup _{|t| \leq \pi}\left|p_{n}(t)\right| \leq C \sqrt{n+1} \quad(n=0,1, \ldots) .
$$


It follows from (6.3) and Lemma 6.3 that $\left\|a_{\left[p_{n}\right]}\right\|_{\ell_{1} \hat{\hat{\otimes}} \ell_{1}} \leq(n+1) C \sqrt{n+1}$. Thus, by (6.1) and (ii),

$$
\left\|a_{\left[p_{n}\right]}\right\|_{\mathcal{M}_{1}}=\sum_{j=0}^{n}(j+1)=(n+1)(n+2) / 2 .
$$

Therefore

$$
\begin{aligned}
h_{n} & \geq \frac{\left\|a_{\left[p_{n}\right]}\right\|_{\mathcal{M}_{l}}}{\left\|a_{\left[p_{n}\right]}\right\|_{\ell_{1} \hat{\hat{\otimes}} \ell_{1}}} \geq \frac{(n+1)(n+2) / 2}{C(n+1)^{3 / 2}} \\
& \geq C_{*} \sqrt{n+1} .
\end{aligned}
$$

REMARK 6.4. We present some examples of sequences of analytic trigonometric polynomials satisfying (i)-(iii).

(a) Let $q_{m}$ for $m=0,1, \ldots$ be the $m$ th Rudin-Shapiro polynomial (cf. [GrM, p. 33]). Then $\operatorname{deg} q_{m}=2^{m}-1$, the $j$ th coefficient of $q_{m}$ equals \pm 1 , and $\sup _{|t| \leq \pi}\left|q_{m}(t)\right|<2^{(m+1) / 2}$. For $n=0,1, \ldots$ let $n+1=2^{m_{1}}+\cdots+2^{m_{k}}$ where $2^{m_{1}} \leq n \leq 2^{m_{1}+1}-2$ and $m_{1}>\cdots>m_{k} \geq 0$. Put

$$
p_{n}(t)=q_{m_{1}}(t)+\sum_{j=1}^{r} q_{m_{j+1}} \exp \left(\sum_{r=1}^{j} 2^{m_{r}} i t\right) .
$$

Then $\operatorname{deg} p_{n}=n$ and

$$
\sup _{|t| \leq \pi}\left|p_{n}(t)\right| \leq \sum_{j=0}^{m_{k}} 2^{\left(m_{k}-j+1\right) / 2}<C \sqrt{n+1}
$$

with some $C \leq 2+\sqrt{2}$.

(b) The van der Corput polynomials (cf. [Z, Ch. V, Theorem 4.7]) are defined by

$$
p_{0}=1, \quad p_{n}=1+\sum_{j=1}^{n} \exp (i j c \ln j+i j x) \quad(n=1,2, \ldots), c>0 \text { fixed. }
$$

The sequence constructed from the Rudin-Shapiro polynomials has real coefficients, while the van der Corput polynomials have complex coefficients. The latter have been used in a different way in [EL] to solve Mazur's problem.

(c) For polynomials with complex coefficients one can make the constant $C$ appearing in (6.3) for large $n$ arbitrarily close to 1 . This follows from the following deep result due to J.-P. Kahane [K]:

There exists a sequence

$$
K_{n}(t)=\sum_{j=1}^{n} a_{j}^{(n)} \exp (i j t), \quad\left|a_{j}^{(n)}\right|=1 \quad(j=1, \ldots, n ; n=1,2, \ldots),
$$


and a sequence $\left(\varepsilon_{n}\right)$ of positive numbers with $\varepsilon_{n}=O\left(n^{-1 / 17} \sqrt{\ln n}\right)$ such that

$$
\left(1-\varepsilon_{n}\right) \sqrt{n} \leq K_{n}(t) \leq\left(1+\varepsilon_{n}\right) \sqrt{n} \quad(|t| \leq \pi ; n=1,2, \ldots) .
$$

REMARK 6.5. In fact, $h_{n}=O(\sqrt{n})$. The rate of growth is the same as for the analogous quantity $u_{n}$ for all matrices in $M_{n}$. Precisely let

$$
u_{n}=\sup _{a \in M_{n} ;\|a\|_{\ell_{1} \hat{\otimes} \ell_{1}}=1}\|a\|_{\mathcal{M}_{1}} \text {. }
$$

Obviously $h_{n} \leq u_{n}$ for all $n$. The next fact is well known and essentially goes back to Orlicz $[\mathrm{O}]$.

\section{Proposition 6.6.}

$$
u_{n} \leq \widetilde{C} \sqrt{n+1} \quad(n=0,1, \ldots),
$$

where

$$
\widetilde{C}= \begin{cases}\sqrt{2} & \text { for real scalars, } \\ 2 / \sqrt{\pi} & \text { for complex scalars } .\end{cases}
$$

Proof. Let $a \in M_{n}$. Let $\left(\eta_{k}\right)$ be either a sequence of independent Bernoulli random variables (real case), or Steinhaus r.v. (complex case). Using the Cauchy-Schwarz inequality and then the Khinchin type inequality we get

$$
\begin{aligned}
\|a\|_{\mathcal{M}_{1}} & \leq \sum_{j=0}^{n} \sqrt{n+1}\left(\sum_{k=0}^{n}|a(j, k)|^{2}\right)^{1 / 2} \\
& \leq \sum_{j=0}^{n} \widetilde{C} \sqrt{n+1} E\left|\sum_{k=0}^{n} \eta_{k} a(j, k)\right| \\
& \leq \widetilde{C} \sqrt{n+1}\|a\|_{\ell_{1} \hat{\hat{\otimes}} \ell_{1}},
\end{aligned}
$$

which proves (6.4). To get (6.5), we use the results of Szarek for Bernoulli r.v. and of Sawa for Steinhaus r.v. on the best constants in Khinchin type inequalities (cf. $[\mathrm{KnKw}]$ and references there).

Our next result strengthens the negative answer to Mazur's Problem 88. It is "almost sharp".

Define the "critical broken line" $\Psi:(0 ; \infty) \rightarrow(-1 ; \infty)$ by

$$
\Psi(t)= \begin{cases}\frac{3}{2} t-1 & \text { for } 0<t \leq 2 \\ t & \text { for } t>2\end{cases}
$$

For $a \in \mathcal{H} \cap \ell_{1} \hat{\hat{\otimes}} \ell_{1}$ put

$$
v_{k}=|a(0, k)| \quad\left(k \in \mathbb{Z}_{+}\right), \quad A_{t}(a)=\sum_{k \in \mathbb{Z}_{+}} v_{k}^{t}(k+1)^{\Psi(t)} \quad(0<t<\infty) .
$$


Theorem 6.7.

(I) If $\beta<\Psi(t)$ then

$$
\sum_{k \in \mathbb{Z}_{+}} v_{k}^{t}(k+1)^{\beta}<\infty \quad \text { for all } a \in \mathcal{H} \cap \ell_{1} \hat{\hat{\otimes}} \ell_{1} .
$$

(II) If $\beta>\Psi(t)$ then

$$
\sum_{k \in \mathbb{Z}_{+}} v_{k}^{t}(k+1)^{\beta}=\infty \quad \text { for some } a \in \mathcal{H} \cap \ell_{1} \hat{\hat{\otimes}} \ell_{1} .
$$

(III) If $4 / 3 \leq t<\infty$ then $A_{t}(a)<\infty$ for all $a \in \mathcal{H} \cap \ell_{1} \hat{\hat{\otimes}} \ell_{1}$.

To prove (I) we need

Lemma 6.8. Let $\widetilde{C}$ be defined by (6.5). Then for $a \in \mathcal{H} \cap \ell_{1} \hat{\otimes} \ell_{1}$,

$$
\sum_{k=2^{m-1}}^{2^{m}-1} v_{k}^{t}(k+1)^{\Psi(t)} \leq\left(2^{3 / 2} \widetilde{C}\|a\|_{\ell_{1} \hat{\hat{\otimes}} \ell_{1}}\right)^{t} \quad(m=1,2, \ldots ; 0<t<\infty) .
$$

Proof. CASe 1: $0 \leq t \leq 2$. The Hölder inequality for the exponent $2 / t$ yields

$$
\sum_{k=2^{m-1}}^{2^{m}-1} v_{k}^{t}(k+1)^{\Psi(t)} \leq 2 \cdot 2^{\Psi(t) m} 2^{(1-t / 2)(m-1)}\left(\sum_{k=2^{m-1}}^{2^{m}-1} v_{k}^{2}\right)^{t / 2},
$$

because if $2^{m-1} \leq k \leq 2^{m}-1$, then

$$
(k+1)^{\Psi(t)} \leq \begin{cases}2^{\Psi(t) m} & \text { for } t \geq 2 / 3 \\ 2 \cdot 2^{\Psi(t) m} & \text { for } 0 \leq t<2 / 3\end{cases}
$$

Recall Littlewood's inequality $[\mathrm{L}]$ and its consequence for Hankel matrices:

Thus,

$$
\begin{aligned}
\sum_{j \in \mathbb{Z}_{+}}\left(\sum_{k \in \mathbb{Z}_{+}}|a(j, k)|^{2}\right)^{1 / 2} \leq \widetilde{C}\|a\|_{\ell_{1} \hat{\hat{\otimes}} \ell_{1}} \quad(a \in \mathcal{M}), \\
\sum_{j \in \mathbb{Z}_{+}}\left(\sum_{k=j}^{\infty} v_{k}^{2}\right)^{1 / 2} \leq \widetilde{C}\|a\|_{\ell_{1} \hat{\hat{\otimes}} \ell_{1}} \quad(a \in \mathcal{H}) .
\end{aligned}
$$

$$
2^{m-1}\left(\sum_{k=2^{m-1}}^{2^{m}-1} v_{k}^{2}\right)^{1 / 2}=\sum_{j=0}^{2^{m-1}-1}\left(\sum_{k=2^{m-1}-j}^{2^{m}-1-j} v_{k+j}^{2}\right)^{1 / 2} \leq \widetilde{C}\|a\|_{\ell_{1} \hat{\otimes} \ell_{1}} .
$$

Since $\Psi(t)=\frac{3}{2} t-1$ for $0<t \leq 2$, (6.8) combined with (6.6) yields

$$
\begin{aligned}
\sum_{k=2^{m-1}}^{2^{m}-1} v_{k}^{t}(k+1)^{\Psi(t)} & \leq 2 \cdot 2^{\Psi(t) m} 2^{(1-t / 2)(m-1)}\left(2^{1-m} \widetilde{C}\|a\|_{\ell_{1} \hat{\dot{\otimes}} \ell_{1}}\right)^{t} \\
& =\left(2^{3 / 2} \widetilde{C}\left\|a_{\ell_{1} \hat{\dot{\otimes}} \ell_{1}}\right\|\right)^{t} .
\end{aligned}
$$

This completes the proof of Lemma 6.8 in Case 1. 
CAse 2: $2<t<\infty$. Since $\Psi(2)=2$, applying Case 1 for $t=2$ we get

$$
\sum_{k=2^{m-1}}^{2^{m}-1} v_{k}^{t}(k+1)^{\Psi(t)} \leq\left(\sum_{k=2^{m-1}}^{2^{m}-1} v_{k}^{2}(k+1)^{2}\right)^{t / 2} \leq\left(2^{3 / 2} \widetilde{C}\|a\|_{\ell_{1} \hat{\otimes} \ell_{1}}\right)^{t}
$$

Proof of Theorem 6.\%. For (I) note that if $\beta-\Psi(t)<0$ then for $m=$ $1,2, \ldots$,

$$
\begin{aligned}
\sum_{k=2^{m-1}}^{2^{m}-1} v_{k}^{t}(k+1)^{\beta} & \leq 2^{(m-1)(\beta-\Psi(t))} \sum_{k=2^{m-1}}^{2^{m}-1} v_{k}^{t}(k+1)^{\Psi(t)} \\
& \leq 2^{(m-1)(\beta-\Psi(t))}\left(2^{3 / 2} \widetilde{C}\|a\|_{\ell_{1} \hat{\hat{\otimes}} \ell_{1}}\right)^{t}
\end{aligned}
$$

Thus

$$
\sum_{k \in \mathbb{Z}_{+}}(k+1)^{\beta} v_{k}^{t}=v_{0}^{t}+\sum_{m=1}^{\infty} \sum_{k=2^{m-1}}^{2^{m}-1} v_{k}^{t}(k+1)^{\beta}<\infty
$$

To prove (II) observe that, by the Banach-Steinhaus principle, it suffices to exhibit a sequence $\left(a_{n}\right) \subset \mathcal{H} \cap \ell_{1} \hat{\otimes} \ell_{1} \backslash\{0\}$ for $n \in \mathbb{Z}_{+}$such that (6.9) $\quad \lim _{n}\left(\left\|a_{n}\right\|_{\ell_{1} \hat{\otimes} \ell_{1}}\right)^{-t} \sum_{k \in \mathbb{Z}_{+}}\left|a_{n}(0, k)\right|^{t}(k+1)^{\beta}=\infty \quad$ whenever $\beta>\Psi(t)$.

Let $0<t \leq 2$. Put $a_{n}=a_{\left[p_{n}\right]}$ for $n \in \mathbb{Z}_{+}$, where $\left(p_{n}\right)$ is any sequence of trigonometric polynomials described in Remark 6.4. Then for $\beta>\Psi(t)>-1$ there is $C=C(\beta)>0$ such that

$$
\sum_{k \in \mathbb{Z}_{+}}\left|a_{\left[p_{n}\right]}(0, k)\right|^{t}(k+1)^{\beta}=\sum_{k=0}^{n}(k+1)^{\beta} \geq C n^{\beta+1} \quad\left(n \in \mathbb{Z}_{+}\right),
$$

while it follows from the proof of Proposition 6.2 that $\left\|a_{\left[p_{n}\right]}\right\|_{\ell_{1} \hat{\hat{\otimes}} \ell_{1}}^{t}=O\left(n^{3 t / 2}\right)$. Thus we get (6.9) because if $\beta>\Psi(t)$ then $n^{\beta+1}>n^{3 t / 2}$.

If $t>2$ we define $a_{n} \in \mathcal{H}$ for $n \in \mathbb{Z}_{+}$by

$$
a_{n}(j, k)= \begin{cases}1 & \text { for } j+k=n \\ 0 & \text { otherwise. }\end{cases}
$$

Then $\left\|a_{n}\right\|_{\ell_{1} \hat{\hat{\otimes}} \ell_{1}}^{t}=(n+1)^{t}$ while $\sum_{k \in \mathbb{Z}_{+}}\left|a_{n}(k, 0)\right|^{t}(k+1)^{\beta}=(n+1)^{\beta}$, which yields (6.9) because if $t>2$ then $\beta>\Psi(t)=t$.

To prove (III) first observe that, by a result due to Littlewood [L], if $a \in \mathcal{M}$ and $\|a\|_{\ell_{1} \hat{\hat{\otimes}} \ell_{1}}<\infty$ then $\sum_{(j, k) \in \mathbb{Z}_{+} \times \mathbb{Z}_{+}}|a(j, k)|^{4 / 3}<\infty$. In particular, if $a \in \mathcal{H} \cap \ell_{1} \hat{\hat{\otimes}} \ell_{1}$, then $A_{4 / 3}(a)=\sum_{k \in \mathbb{Z}_{+}} v_{k}^{4 / 3}(k+1)<\infty$. 
Next, consider the case $t=2$. Then $\Psi(2)=2$. We have to prove that if $a \in \mathcal{H} \cap \ell_{1} \hat{\otimes} \ell_{1}$, then $A_{2}(a)=\sum_{k=0}^{\infty} v_{k}^{2}(k+1)^{2}<\infty$. Put

$$
V_{k}=\left(\sum_{\nu=k}^{\infty} v_{\nu}^{2}\right)^{1 / 2} \quad(k=0,1, \ldots) .
$$

Note that

$$
V_{k} V_{p} \geq V_{p}^{2}=\sum_{\nu=p}^{\infty} v_{\nu}^{2} \quad(p=k, k+1, \ldots ; k=0,1, \ldots) .
$$

Clearly we can write (6.7) as $\widetilde{C}\|a\|_{\ell_{1} \hat{\hat{\otimes}} \ell_{1}} \geq \sum_{k \in \mathbb{Z}_{+}} V_{k}$. Squaring both sides, we have

$$
\begin{aligned}
\widetilde{C}^{2}\|a\|_{\ell_{1} \hat{\hat{\theta}} \ell_{1}}^{2} & \geq\left(\sum_{k \in \mathbb{Z}_{+}} V_{k}\right)^{2}=\sum_{k \in \mathbb{Z}_{+}}\left(V_{k}^{2}+2 \sum_{p=k+1}^{\infty} V_{k} V_{p}\right) \\
& \geq \sum_{k \in \mathbb{Z}_{+}}\left(V_{k}^{2}+2 \sum_{p=k+1}^{\infty} V_{p}^{2}\right)=\sum_{k \in \mathbb{Z}_{+}} \sum_{\nu=0}^{k}(2 \nu+1) v_{k}^{2}=A_{2}(a) .
\end{aligned}
$$

Next, let $4 / 3<t<2$. Choose $\theta$ so that $t=\theta \cdot 4 / 3+2(1-\theta)$. Then $0<\theta<1$. The numerical inequality $\alpha+\beta \geq \alpha^{\theta} \beta^{1-\theta}$ for $\alpha, \beta>0$ implies

$$
\begin{aligned}
A_{4 / 3}(a)+A_{2}(a) & =\sum_{k \in \mathbb{Z}_{+}}\left(v_{k}^{4 / 3}(k+1)+v_{k}^{2}(k+1)^{2}\right) \\
& \geq \sum_{k \in \mathbb{Z}_{+}} v_{k}^{\theta \cdot 4 / 3+2(1-\theta)}(k+1)^{\theta+2(1-\theta)}=A_{t}(a) .
\end{aligned}
$$

Finally, if $t>2$, then

$$
A_{t}(a)=\sum_{k \in \mathbb{Z}_{+}} v_{k}^{t}(k+1)^{t} \leq\left(\sum_{k \in \mathbb{Z}_{+}} v_{k}^{2}(k+1)^{2}\right)^{t / 2}=A_{2}(a)^{t / 2}
$$

REMARK 6.9. The behavior of the series $A_{t}(a)=\sum_{k \in \mathbb{Z}_{+}} v_{k}^{t}(k+1)^{\Psi(t)}$ seems to be unknown for $0<t<4 / 3$. Since the harmonic series diverges, $\lim _{t \rightarrow 0} A_{t}(a)=\infty$ for any $a \in \mathcal{H} \cap \ell_{1} \hat{\hat{\otimes}} \ell_{1}$ whose symbol has almost all coefficients different from 0 .

REMARK 6.10.

(i) If $a \in \mathcal{M}$ and $\|a\|_{\ell_{1} \hat{\hat{\otimes}} \ell_{1}}<\infty$ then $a \in \mathcal{S}_{1}$ and $\|a\|_{1} \leq\|a\|_{\ell_{1} \hat{\hat{\otimes}} \ell_{1}}$.

(ii) There exists $a \in \mathcal{S}_{1} \cap \mathcal{H}$ with $\|a\|_{\ell_{1} \hat{\hat{\otimes}} \ell_{1}}=\infty$.

Proof. Part (i) is known. Let $b \in \mathcal{M}_{0}$. Clearly $\sum_{t \in \mathbb{Z}_{+}}|b(t, k)|^{2} \leq\|b\|_{B\left(\ell_{2}\right)}$ for $k \in \mathbb{Z}_{+}$. Thus, by the Schwarz inequality and by (6.7), 


$$
\begin{aligned}
|\operatorname{Tr}(a b)| & =\left|\sum_{k \in \mathbb{Z}_{+}} \sum_{s \in \mathbb{Z}_{+}} a(s, k) b(k, s)\right| \\
& \leq \sum_{k \in \mathbb{Z}_{+}}\left(\sum_{s \in \mathbb{Z}_{+}}|a(s, k)|^{2}\right)^{1 / 2}\left(\sum_{t \in \mathbb{Z}_{+}}|b(k, t)|^{2}\right)^{1 / 2} \\
& \leq \sum_{k \in \mathbb{Z}_{+}}\left(\sum_{s \in \mathbb{Z}_{+}}|a(s, k)|^{2}\right)^{1 / 2}\|b\|_{B\left(\ell_{2}\right)} \leq \widetilde{C}\|a\|_{\ell_{1} \hat{\hat{\otimes}} \ell_{1}}\|b\|_{B\left(\ell_{2}\right)} .
\end{aligned}
$$

Since $\mathcal{M}_{0}$ is norm dense in $K\left(\ell_{2}\right)$, the trace duality between $K\left(\ell_{2}\right)$ and $\mathcal{S}_{1}$ implies that $\|a\|_{1} \leq\|a\|_{\ell_{1} \hat{\hat{\otimes}} \ell_{1}}$.

(ii) It is enough to show

$$
\sup _{a \in \mathcal{M}_{0} \cap \mathcal{H} ;\|a\|_{1} \leq 1}\|a\|_{\ell_{1} \hat{\hat{\otimes}} \ell_{1}}=\infty .
$$

We need [Pe, Ch. VI, Lemma 1.3]: If $F$ is an analytic trigonometric polynomial of degree $n$ then

$$
\left\|a_{[F]}\right\|_{1} \leq(n+1) \frac{1}{2 \pi} \int_{-\pi}^{\pi}|F(t)| d t
$$

Let $F_{n}$ be the $n$-shifted Fejér kernel,

$$
F_{n}=\frac{1}{n+1}\left[\sum_{k=0}^{n}(k+1) \exp (i k)+\sum_{k=n+1}^{2 n}(2 n+1-k) \exp (i k)\right] \quad(n=1,2, \ldots) .
$$

Peller's lemma mentioned above and properties of Fejér kernels imply that $\left\|\frac{1}{2 n+1} a_{\left[F_{n}\right]}\right\|_{1}=1$. Since the non-zero coefficients of $F_{n}$ are positive, we have $\left\|\frac{1}{2 n+1} a_{\left[F_{n}\right]}\right\|_{\ell_{1} \hat{\hat{\theta}} \ell_{1}}=\frac{1}{2 n+1} \sum_{j=0}^{2 n} \sum_{k=0}^{2 n-j} a_{\left[F_{n}\right]}(j, k)=\frac{n+1}{2} \quad(n=1,2, \ldots)$.

Next we consider Problem 8 of [Scb]. Let $\widetilde{B}: c \times c \rightarrow c$ be the bilinear form defined by

$$
\widetilde{B}\left(\left(x_{n}\right),\left(y_{n}\right)\right)=\left(z_{n}\right) \quad \text { where } z_{n}=(n+1)^{-1} \sum_{k=0}^{n} x_{k} y_{n-k} \quad\left(n \in \mathbb{Z}_{+}\right),
$$

where $c$ stands for the space of all convergent scalar sequences. Stanisław Mazur asked: "Does $\widetilde{B}$ map $c \times c$ onto $c$ ?". The answer is negative:

Proposition 6.11. $\widetilde{B}$ is not surjective.

Proof. Recall that every bounded bilinear form $\widetilde{A}: X \times Y \rightarrow Z$, where $X, Y, Z$ are Banach spaces, uniquely extends to a linear operator $A$ : $X \hat{\otimes} Y \rightarrow Z$ (here $\hat{\otimes}$ denotes the projective tensor product) such that

$$
\widetilde{A}(x, y)=A(x \otimes y) \quad(x \in X, y \in Y) ;
$$

moreover $\|\widetilde{A}\|=\|A\|$ (cf. [DeF, Ch. I, §3]).

Clearly if $\widetilde{A}$ is surjective then so is $A$. 
By [DnSc, Ch. VI, §6], if $A: V \rightarrow Z$ ( $V, Z$ Banach spaces) is surjective then the adjoint operator $A^{*}: Z^{*} \rightarrow V^{*}$ is an isomorphic embedding, equivalently

$$
\left\|A^{*}\left(z^{*}\right)\right\| \geq \gamma\left\|z^{*}\right\| \quad\left(z^{*} \in Z^{*}\right) \text { for some } \gamma>0 .
$$

We show that the linearization $B$ of Mazur's bilinear form $\widetilde{B}$ defined by (6.10) violates (6.11).

Clearly $\|B\|=\left\|B^{*}\right\|=\|\widetilde{B}\|=1$. Identify $c^{*}$ with $\ell_{1}\left(\mathbb{Z}_{+} \cup\{\infty\}\right)$. Let $\left(\delta_{k}^{*}\right)_{k \in \mathbb{Z}_{+} \cup\{\infty\}}$ be the unit vector basis of $c^{*}$. An easy calculation gives

$$
B^{*}\left(\delta_{k}^{*}\right)(x \otimes y)=\frac{1}{k+1} \sum_{j=0}^{k} x_{j} y_{k-j} \quad\left(x=\left(x_{n}\right) \in c, y=\left(y_{n}\right) \in c ; k \in \mathbb{Z}_{+}\right) .
$$

Put $E_{n}=\operatorname{span}\left\{\delta_{k}^{*}: 0 \leq k \leq n\right.$ for $\left.n=0,1,2, \ldots\right\}$ and let

$$
\gamma_{n}=\inf _{0 \neq x^{*} \in E_{n}} \frac{\left\|B^{*} x^{*}\right\|_{(c \hat{\otimes} c)^{*}}}{\left\|x^{*}\right\|_{c^{*}}} \quad(n=0,1, \ldots) .
$$

Let $\left(p_{n}\right)_{n \in \mathbb{Z}_{+}}$be a sequence of analytic trigonometric polynomials satisfying (i)-(iii) in the proof of Proposition 6.2. Fix $n \in \mathbb{Z}_{+}$. Consider the functional $\psi$ on $c \hat{\otimes} c$ defined by

$$
\psi=B^{*}\left(\sum_{k=0}^{n}(k+1) a_{\left[p_{n}\right]}(0, k) \delta_{k}^{*}\right) .
$$

Then, remembering that $\operatorname{deg} p_{n}=n$ and $a_{\left[p_{n}\right]}(j, k)=0$ for $j+k>n$, we have

$$
\begin{aligned}
\|\psi\|_{(c \hat{\otimes} c)^{*}} & =\sup _{\|x\|_{c} \leq 1 ;\|y\|_{c} \leq 1}|\psi(x \otimes y)| \\
& =\sup _{\|x\|_{c} \leq 1 ;\|y\|_{c} \leq 1}\left|\sum_{k=0}^{n} \sum_{j+k=n} a_{\left[p_{n}\right]}(0, j+k) x_{j} y_{k}\right| \\
& =\sup _{\|x\|_{c} \leq 1 ;\|y\|_{c} \leq 1}\left|\sum_{j=0}^{n} \sum_{k=0}^{n} a_{\left[p_{n}\right]}(j, k) x_{j} y_{k}\right|=\left\|a_{\left[p_{n}\right]}\right\|_{\ell_{1} \hat{\dot{\otimes}} \ell_{1}} .
\end{aligned}
$$

Thus, by (6.2) and (6.3), we have $\|\psi\|_{(c \hat{\otimes} c)^{*}} \leq C(n+1)^{3 / 2}$. Similarly, invoking (ii) gives

$$
\left\|\sum_{k=0}^{n}(k+1)\left|a_{\left[p_{n}\right]}(0, k)\right| \delta_{k}^{*}\right\|_{c^{*}}=\sum_{k=0}^{n}(k+1)\left|a_{\left[p_{n}\right]}(0, k)\right|=\frac{(n+1)(n+2)}{2} .
$$

Thus, appealing to the definition of $\gamma_{n}$, we obtain

$$
\left.C(n+1)^{3 / 2} \geq\|\psi\|_{(c \hat{\otimes} c)^{*}} \geq \gamma_{n} \| \sum_{k=0}^{n}(k+1) a_{\left[p_{n}\right]}(0, k) \delta_{k}^{*}\right) \|_{c^{*}}=\gamma_{n} \frac{(n+1)(n+2)}{2} .
$$

Therefore $\gamma_{n}=O\left(n^{-1 / 2}\right)$; in particular $\lim _{n} \gamma_{n}=0$. Thus $B$ violates (6.11). 
REMARK 6.12. The same proof shows that $\widetilde{B}\left(c_{0} \times c_{0}\right) \neq c_{0}$. Clearly $\widetilde{B}\left(c_{0} \times c_{0}\right) \supset c_{0}$.

REMARK 6.13. Recall (cf. [Pi 3, p. 61], [MiP]) that the $n$th Gelfand number of an operator $T: V \rightarrow Z$ is defined by

$$
c_{n}(T)=\inf \left\{\left\|T_{\mid W}\right\|: W \subset V, \operatorname{codim} W<n\right\} .
$$

The Gelfand numbers measure "non-surjectivity" of $T$. Our proof of 6.11 combined with a standard duality argument shows that $c_{n}(B) \leq \gamma_{n-1}$. Hence $c_{n}(B)=O\left(n^{-1 / 2}\right)$. It is not hard to show that this is sharp, i.e. there is $C^{\prime}>0$ such that $c_{n}(B) \geq C^{\prime} n^{-1 / 2}$ for all $n$.

\section{References}

[A] W. Arveson, Interpolation problems in nest algebras, J. Funct. Anal. 20 (1975), 208-233.

[Bn] G. Bennett, Schur multipliers, Duke J. Math. 44 (1977), 603-631.

[BP] O. Blasco and A. Pełczyński, Theorems of Hardy and Paley for vector-valued analytic functions and related classes of Banach spaces, Trans. Amer. Math. Soc. 333 (1991), 331-367.

[DLuQ] M. Déchamps-Gondim, F. Lust-Piquard and H. Queffélec, On the minorant properties in $C_{p}(H)$, Pacific J. Math. 119 (1985), 89-101.

[DeF] A. Defant and K. Floret, Tensor Norms and Operator Ideals, North-Holland Math. Stud. 176, North-Holland, Amsterdam, 1993.

[DoS] P. Dodds and F. Sukochev, RUC-decompositions in symmetric operator spaces, Integral Equations Operator Theory 29 (1997), 269-287.

[Dg] R. G. Douglas, Banach Algebra Techniques in Operator Theory, Academic Press, New York, 1972.

[DnSc] N. Dunford and J. T. Schwartz, Linear Operators I, Interscience, New York, 1958.

[Du] P. L. Duren, Theory of $H_{p}$ Spaces, Academic Press, New York, 1970.

[EL] P. P. B. Eggermont and Y. J. Leung, On a factorization problem for convergent sequences and on Hankel forms in bounded sequences, Proc. Amer. Math. Soc. 96 (1986), 269-274.

[GK] I. C. Gohberg and M. G. Krĕn, Theory and Applications of Volterra Operators in Hilbert Spaces, Transl. Math. Monogr. 24, Amer. Math. Soc., Providence, RI, 1970.

[GrM] C. C. Graham and O. C. McGehee, Essays in Commutative Harmonic Analysis, Grundlehren Math. Wiss. 238, Springer, Berlin, 1979.

[HL] G. H. Hardy and J. E. Littlewood, Theorems concerning mean values of analytic or harmonic functions, Quart. J. Math. Oxford Ser. 12 (1941), 221-256.

[K] J.-P. Kahane, Sur les polynômes à coefficients unimodulaires, Bull. London Math. Soc. 12 (1980), 321-342.

[KKL] J.-P. Kahane, Y. Katznelson et K. de Leeuw, Sur les coefficients de Fourier des fonctions continues, C. R. Acad. Sci. Paris Sér. I 285 (1977), 1001-1004.

[KnKw] H. König and S. Kwapień, Best Khintchine type inequalities for sums of independent random variables, Positivity 5 (2001), 115-152. 
[KwP] S. Kwapień and A. Pełczyński, On two problems of S. Mazur from the Scottish $B o o k$, lecture at the Colloquium dedicated to the memory of Stanisław Mazur, Warsaw Univ., 1985 (unpublished).

[L] J. E. Littlewood, On bounded bilinear forms in an infinite number of variables, Quart. J. Math. 1 (1930), 164-174.

[Lu] F. Lust-Piquard, On the coefficient problem: a version of the Kahane-Katznelson-de Leeuw theorem for spaces of matrices, J. Funct. Anal. 149 (1997), 352376 .

[M] B. Maurey, Tout opérateur d'une $C^{*}$-algèbre dans un espace de cotype 2 se factorise par un Hilbert, d'après G. Pisier, Séminaire sur la géométrie des espaces de Banach, 1975-1976, École Polytechnique, exp. XXI.

[MiP] B. S. Mityagin and A. Pełczyński, Nuclear operators and approximative dimension, in: Proc ICM Moscow, 1966, 366-372.

[N] F. Nazarov, The Bang solution to the coefficient problem, Algebra i Analiz 9 (1987), 272-287 (in Russian).

[O] W. Orlicz, Über unbedingte Konvergenz in Funktionenräumen, Studia Math. 4 (1934), 33-37.

[Ov] V. I. Ovchinnikov, Noncommutative BMO spaces, coherent nuclearity, and bounded extensions of matrices, Dokl. Akad. Nauk 363 (1998), 17-19 (Russian).

[Pa 1] R. E. A. C. Paley, On the lacunary coefficients of power series, Ann. of Math. 34 (1936), 615-616.

[Pa 2] - A note on power series, J. London Math. Soc. 7 (1932), 122-130.

[Pe] V. V. Peller, Hankel Operators and Their Applications, Springer Monogr. Math., Springer, Berlin, 2003.

[Pi 1] G. Pisier, Grothendieck's theorem for non-commutative $C^{*}$-algebras, with an appendix on Grothendieck's constant, J. Funct. Anal. 29 (1978), 397-415.

[Pi 2] - Interpolation between $H^{p}$-spaces and non-commutative generalizations. I, Pacific J. Math. 155 (1992), 341-368.

[Pi 3] - The Volume of Convex Bodies and Banach Space Geometry, Cambridge Tracts Math. 94, Cambridge Univ. Press, Cambridge, 1989.

[PiX] G. Pisier and Q. Xu, Non-commutative $L_{p}$-spaces, in: Handbook of the Geometry of Banach Spaces, Vol. II, Elsevier, Amsterdam, 2003, 1459-1517.

[Scb] The Scottish Book, R. D. Mauldin (ed.), Birkhäuser, Boston, MA, 1979.

[Sh] A. L. Shields, An analogue of a Hardy-Littlewood-Fejér inequality for upper triangular trace class operators, Math. Z. 182 (1983), 473-484.

[SlSt] W. T. Sledd and D. A. Stegenga, An $H^{1}$ multiplier theorem, Ark. Mat. 19 (1981), 265-270.

[SzW] S. J. Szarek and T. Wolniewicz, A proof of Fefferman's theorem on multipliers, Inst. Math., Polish Acad. Sci., Preprint No. 209 (1980).

[Z] A. Zygmund, Trigonometric Series, Vol. I, 2nd ed., Cambridge Univ. Press, Cambridge, 1959.

Institute of Mathematics

Polish Academy of Sciences

Śniadeckich 8, 00-956 Warszawa, Poland

E-mail: olek@impan.gov.pl
School of Informatics and Engineering Flinders University of South Australia 5042 Bedford Park, Australia E-mail: sukochev@infoeng.flinders.edu.au 